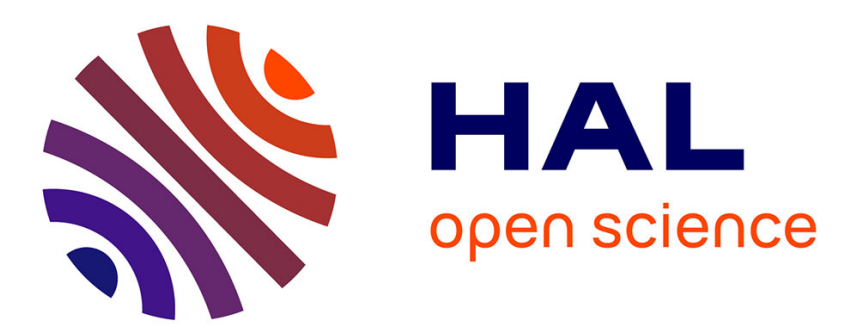

\title{
Uncertainty Quantification for Fatigue Life of Offshore Wind Turbine Structure
}

Abraham Nispel, Stephen Ekwaro-Osire, João Paulo Dias, Americo Cunha Jr

\section{To cite this version:}

Abraham Nispel, Stephen Ekwaro-Osire, João Paulo Dias, Americo Cunha Jr. Uncertainty Quantification for Fatigue Life of Offshore Wind Turbine Structure. ASCE-ASME Journal of Risk and Uncertainty in Engineering Systems, Part B: Mechanical Engineering, 2021, 7, pp.040901. 10.1115/1.4051162 . hal-03287896

\section{HAL Id: hal-03287896 https://hal.science/hal-03287896}

Submitted on 16 Jul 2021

HAL is a multi-disciplinary open access archive for the deposit and dissemination of scientific research documents, whether they are published or not. The documents may come from teaching and research institutions in France or abroad, or from public or private research centers.
L'archive ouverte pluridisciplinaire HAL, est destinée au dépôt et à la diffusion de documents scientifiques de niveau recherche, publiés ou non, émanant des établissements d'enseignement et de recherche français ou étrangers, des laboratoires publics ou privés. 


\section{Abraham Nispel \\ Mem. ASME \\ Department of Mechanical Engineering, \\ Texas Tech University, \\ Lubbock, TX 79409 \\ e-mail: abraham.nispe|@ttu.edu}

Stephen Ekwaro-0sire ${ }^{1}$

Fellow ASME

Department of Mechanical Engineering,

Texas Tech University,

Lubbock, TX 79409

e-mail: stephen.ekwaro-osire@ttu.edu

\section{João Paulo Dias}

Mem. ASME

Department of Civil and Mechanical Engineering,

Shippensburg University of Pennsylvania,

Shippensburg, PA 17257

e-mail: jpdias@ship.edu

Americo Cunha, Jr.

Institute of Mathematics and Statistics, Rio de Janeiro State University,

Rio de Janeiro, RJ 20550-900, Brazil

e-mail: americo.cunha@uerj.br

\section{Uncertainty Quantification for Fatigue Life of Offshore Wind Turbine Structure}

This study aims to address the question: can the structural reliability of an offshore wind turbine (OWT) under fatigue loading conditions be predicted more consistently? To respond to that question this study addresses the following specific aims: (1) to obtain a systematic approach that takes into consideration the amount of information available for the uncertainty modeling of the model input parameters and (2) to determine the impact of the most sensitive input parameters on the structural reliability of the OWT through a surrogate model. First, a coupled model to determine the fatigue life of the support structure considering the soil-structure interaction under 15 different loading conditions was developed. Second, a sensitivity scheme using two global analyses was developed to consistently establish the most and least important input parameters of the model. Third, systematic uncertainty quantification (UQ) scheme was employed to model the uncertainties of model input parameters based on their available-data-driven and physics-informed-information. Finally, the impact of the proposed UQ framework on the OWT structural reliability was evaluated through the estimation of the probability of failure of the structure based on the fatigue limit state design criterion. The results show high sensitivity for the wind speed and moderate sensitivity for parameters usually considered as deterministic values in design standards. Additionally, it is shown that applying systematic UQ not only produces a more efficient and better approximation of the fatigue life under uncertainty, but also a more accurate estimation of the structural reliability of offshore wind turbine's structure during conceptual design. Consequently, more reliable, and robust estimations of the structural designs for large offshore wind turbines with limited information may be achieved during the early stages of design.

Keywords: offshore wind turbines, uncertainty quantification, structural reliability, fatigue life, maximum entropy principle

\section{Introduction}

Offshore wind energy is steadily growing due to (1) offshore wind speed is faster and less turbulent than onshore wind speed, (2) offshore wind farms have less space limitation and transport issues associated with the construction and erection of taller turbines in contrast to onshore farms, and (3) offshore wind power is a clean source of energy that contributes to reducing carbon emissions [1,2]. Nevertheless, the structure of offshore wind turbines (OWTs), i.e., tower and foundation, is highly sensitive to dynamic amplification and fatigue damage, as the excitation frequencies resulting from wind and wave loads approach the natural frequency of the structure [3-5]. Whilst Fatigue damage calculations involve several parameters, and also several combinations of environmental conditions to account for realistic operation scenarios, it is also paramount to account for the uncertainty in the models and parameters to ensure a service life of at least 20 years $[4,6]$. Albeit accounting for the variability of certain parameters, such as wind speed and wave height, is a common practice in the industry, other parameters are normally considered as constants, even though data has suggested that they may vary significantly $[7,8]$. Therefore, it is necessary to analyze the importance of those variables in terms of the structural response and reliability of the OWT.

Sensitivity analysis (SA) has received a lot of attention in the field of offshore wind energy due to the need to identify the most significant parameters that affect the design of support structures. However, identifying such parameters is a rather challenging task

${ }^{1}$ Corresponding author. since OWT structural design involves several elements: design criteria, complex nonlinear models, and a large number of inputmodel parameters, which altogether add a significant amount of uncertainty to the sensitivity results [9].

To account for the model uncertainties and nonlinearities in the sensitivity results, researchers across several disciplines suggest the use of global sensitivity analysis (GSA) [10]. GSA aims to assess the most relevant input-model parameters by analyzing the individual and combined effects that the uncertainty of each input parameter has on the total variance of the response. As a result, a complete exploration of design space considering interaction effects among input-model parameters can be analyzed giving more robust sensitivity indices [11]. Given the benefits of GSA, various authors have attempted to apply GSA in the field of OWT structural design. Nevertheless, due to the inconsistency in the selection of the initial set of input-model parameters, and the lack of clear convergence metrics on the results, no substantial conclusions regarding parameters that should be either discarded or maintained (which is also known as model reduction and prioritization) were observed $[9,12]$. To overcome this situation, the utilization of more than one GSA method has been proposed. For example, two efficient methods such as the Morris approach also called the elementary effects (EE) approach and rank regression was applied and showed good agreement in the results [10]. The application of the aforementioned methods, however, is limited due to their lack of robustness to identify the most important parameters, thereby reducing their utilization for model reduction, before using more sophisticated methods [11]. Analysis of variance approaches, i.e., Sobol indices, have been consistently applied in models with noncorrelated inputs, and are usually used as a benchmark for testing other sensitivity approaches [10]. That 
is explained by their capacity to accurately identify (1) noninfluential and influential parameters and (2) their interaction effects. Despite that, their accuracy heavily relies on the number of simulations performed to achieve convergence, thereby making them computationally expensive and prohibited for complex models with a large number of input parameters [13]. To improve the efficiency of the Sobol' approach, new sampling techniques and metamodels (surrogate models) have been suggested; the use of metamodels, however, paired with Monte Carlo (MC) simulation has shown more accurate results than their sampling counterparts [10]. Although metamodels have been widely used for uncertainty quantification (UQ) in OWT structures [14], these models coupled with Sobol' GSA have not been applied to support the design of OWT under fatigue loads yet. Hence, there is a need to develop sensitivity schemes that improve the efficiency and decisionmaking of current sensitivity analyses in the design under fatigue.

As far as reliability is concerned, uncertainties in the loading conditions, soil, and material properties highly influence the fatigue response, and thus the OWT reliability predictions. In most cases, ensuring a certain reliability value has been shown to drive the cost of the structure to around $30 \%$ of the entire OWT because conservative design approaches are often employed to make up for the model input-parameters uncertainties in the model response [5]. Several probabilistic analyses have been conducted using simplified methods to account for model inputparameters uncertainties in a more sophisticated fashion $[1,6,15]$. However, those analyses have failed to systematically account for uncertainties, leading eventually to underestimations or overestimations of the reliability [16].

The quality of probabilistic predictions, constructed with a stochastic model, is closely linked to its physical consistency, as well as its reliability with the known information about the system of interest. Low-quality probabilistic forecasts can be a strong indication that the used probabilistic model is physically inconsistent, either because it does not take into account known information about the system of interest, or possibly because it does not respect the basic physics of the problem. Therefore, there is still a need to develop a robust UQ scheme to assess the uncertainties in the main input parameters of the OWT models, which go in the direction of constructing probabilistic models which are more consistent with the physical constraints of the input parameters.

Based on the above-mentioned issues regarding the selection of the input parameters, uncertainty modeling, and accurate reliability estimation, this study responds to the following question: can the structural reliability of an OWT under fatigue loading be predicted more consistently? The specific aims to address this question are: (1) to obtain a systematic approach that takes into consideration the amount of information available for the uncertainty modeling of the model input parameters and (2) to determine the impact of the most sensitive input parameters on the structural reliability of the OWT through a surrogate model. To achieve the two specific aims, a coupled model, which combines the simulation codes FAST and TurbSim, developed by the National Renewable Energy Laboratories (NREL), the Rainflow counting (RFC) technique, and the Palmgren-Miner rule, was developed to determine the fatigue life of the OWT structure. The probability density functions (PDFs) were determined by developing a systematic UQ scheme. The scheme models the uncertainties of the input parameters based on the type of information available at the moment of the analysis: (1) physics-based information and (2) data-driven information. The most and less sensitive input parameters of the OWT design under fatigue loading were established by conducting a global sensitivity analysis. Finally, the prediction of the structural reliability using a probabilistic model to establish the damage probability distribution under 30 years of service. The rest of the paper is organized as follows: methodology, results, and discussions of the main findings of this work, and finally, conclusions and recommendations for future research on this topic.

\section{Methodology}

In this study, a deterministic model and a UQ framework were developed to investigate the potential inconsistencies in the estimation of the reliability of a $5 \mathrm{MW}$ OWT [17] with a monopile foundation under fatigue loading conditions. The overarching methodology is depicted in Fig. 1. The rest of this section is divided as follows: deterministic modeling, sensitivity analysis, probabilistic modeling, and specification of the analysis.

2.1 Deterministic Modeling. The deterministic model used in this paper is a combination of two models: the first model aims to establish the main characteristics of a monopile foundation accounting for the structural, geotechnical, and environmental complexities with a limited amount of data $[18,19]$. The second is the combination of FAST and TurbSim codes developed by NREL that aims to determine the dynamic response of a $5 \mathrm{MW}$ OWT. The main benefits of using FAST and TurbSim are their capabilities: (1) to simulate realistic random turbulent time series; (2) to account for the rotational sampling effect, and the geometry of the blades by using the corrected blade momentum theory; and (3) to account for the effects of turbine control mechanisms in the below-rated and above-rated power conditions by using two independent control systems (i.e., a generator-torque controller and a full-span rotor-collective blade-pitch controller) [20]. A schematic diagram of the different submodels, i.e., environmental model, loading model, and fatigue model, that comprise the simplified model developed to compute the fatigue damage of the structure, is illustrated in Fig. 2. The details of each model and parameters used in each submodel are introduced in the following Secs. 2.1.1 and 2.1.2.

2.1.1 Environmental and Loading Models. In this section, the methods to simulate random time series for the turbulent wind speed, $u_{H}(t)$, and wave surface elevation, $\eta(t)$, from their respective power spectral densities are explained. On the one hand, TurbSim was used to generate coherent stochastic turbulent 10 minute time series that represent the full-field flow around the turbine rotor. The JONSWAP spectrum, $S_{\eta}(t)$, on the other hand, was used for modeling the stochastic behavior of the waves $[19,21,22]$.

After the stochastic time series were determined, they served as inputs to the loading model, wherein the loads exerted over the structure were assessed. The wind loads, i.e., force and moment, $F_{U}(t)$ and $M_{U}(t)$, at the tower base were determined through the FAST simulations, which not only accounts for the effects of the control system and rotational sampling but also the geometric effects of the blades in the structural response of the turbine. The hydrodynamic load, $F_{W}(t)$, which is the force exerted on the pile

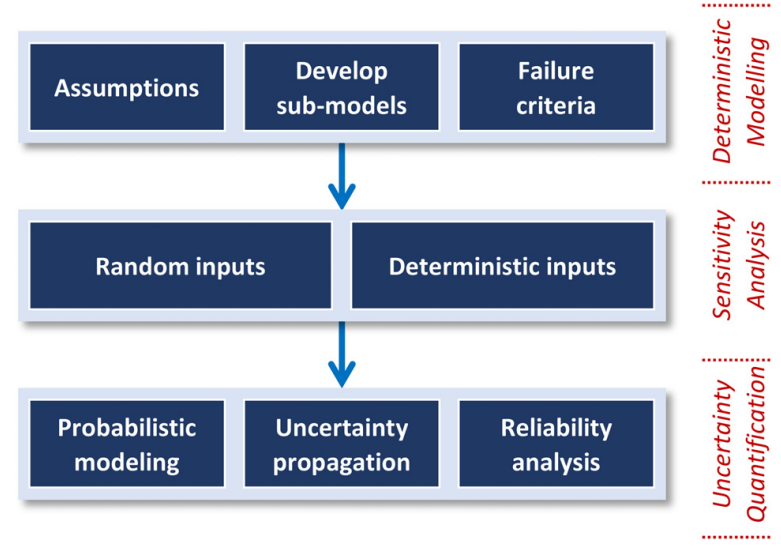

Fig. 1 Schematic representation of the general methodology of analysis employed to access the OWT system 


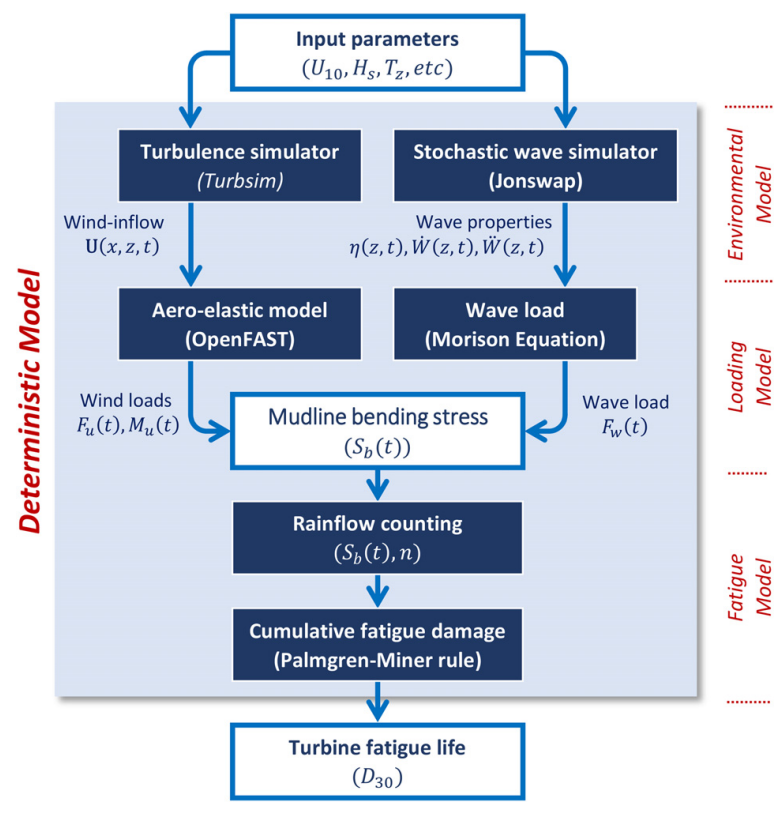

Fig. 2 Schematic representation of the deterministic model for the OWT system

due to the action of the drag and inertia of the waves, was modeled as a point load at the mean sea level (MSL), using Morison's equation

$$
\begin{gathered}
F_{w}(t)=F_{w i}(t)+F_{w d}(t) \\
F_{w i}(t)=\int_{-d w}^{\eta(z)} 0.25 \rho_{s w} c_{m} \pi D_{P}^{2} \ddot{W}(z, t) d z \\
F_{w d}(t)=\int_{-d w}^{\eta(z)} 0.5 \rho_{s w} c_{d} D_{P}|\dot{W}(z, t)| \dot{W}(z, t) d z
\end{gathered}
$$

wherein $F_{w i}$ and $F_{w d}$ are inertial and drag components of the wave loads, $\dot{W}(z, t) \& \ddot{W}(z, t)$, are the wave speed and acceleration computed assuming the Airy wave theory [4-6]. A dynamic amplification factor, DAF, was multiplied to hydrodynamic load and its value was estimated as [22]

$$
\mathrm{DAF}=\frac{1}{\sqrt{\left(1-f_{0}\right)^{2}+\left(2 \zeta f_{0}\right)^{2}}}
$$

where the parameter $\xi$ is the total damping which combines all forms of damping (i.e., aerodynamic, hydrodynamic, and structural), and $f_{0}$ is the natural frequency of the structure. The natural frequency of the structure was determined based on the simplified dynamic soil-structure interaction model, which models the foundation stiffness as a set of nonlinear springs at the mudline based on information about the pile parameters, $D_{P}$ and $t_{P}$, and Young modulus of the soil, $E_{\mathrm{SO}}[18,19]$.

The mudline bending moment was calculated as

$$
M_{s b}(t)=F_{U}(t)+M_{U}(t)+F_{w}(t) d_{w}
$$

where $F_{U}(t)$ and $M_{U}(t)$ are the resulting force and moment generated by the wind loads at the tower base calculated using FAST and TurbSim, $d_{\mathrm{HUB}}$ is the hub height, and $d_{W}$ is the height from the mudline to the MSL (see Fig. 3).

It is important to mention that the mudline bending moment was determined assuming that the wind-wave misalignment either from the onshore or offshore winds is negligible base on the data provided for the area of Blyth-that is from the environmental conditions for the analysis were taken [23]. Despite the low probabilities of wind-wave misalignment for the area used in this study, this assumption may have significant impacts on the structural response of the OWT, in particular for large OWT [24]. As a result, albeit in this study the misalignment happened to be negligible, future studies may consider other locations to study this phenomenon in more detail. Finally, the main characteristics of a $5 \mathrm{MW}$ OWT with a monopile foundation, such as geometrical properties, were summarized in Table 1 [17].

2.1.2 Fatigue Model. The time-variant bending stress at the mudline is computed considering the following expression [25]:

$$
S_{b}(t)=\frac{c M_{s b}(t)}{I_{C M}}
$$

in which $c$ is the horizontal distance from the neutral axis of the pile to point of analysis, $I_{\mathrm{CM}}$ is the moment of inertia around the direction perpendicular to the neutral axis. While frequency domain and time domain methods are suitable to determine the midrange stresses, the time domain is preferable because it is proven to be more accurate than its frequency counterpart; consequently, the RFC method is applied in this study [4]. The RFC transforms the time-variant stress input into several blocks of loadings, $\Delta S_{i}$, also termed midrange stress, and counts the number of cycles, $n_{i}$, that every block was applied to the structure. Thereafter, the total number of cycles to failure of every individual loading block, $N_{i}$, can be found using the bilinear $\mathrm{S}-\mathrm{N}$ curve class $E$ for circular welded joints [26]

$$
N_{i}=10^{\log (a)-m \log \left(\Delta S_{i}(\mathrm{SCF})\right)\left(\frac{t_{p}}{t_{\text {ref }}}\right)^{k}}
$$

wherein $\log (a)$ is the intercept with the $\log (N)$ axis, $t_{p}$ is the pile thickness, $t_{\text {ref }}$ is reference thickness and SCF is the stress intensity factor. The cumulative damage due to the combination of all the stress conditions $\Delta S_{i}$ can be assessed using the Palmgren-Miner rule $[4,21,26]$. In which the total life of the structure is expressed in terms of the damage, $D$, that is calculated as the ratio between $n_{i}$ and $N_{i}$, so when $D$ is equal to one the structure fails

$$
D=\sum_{i=1}^{E_{s}} \frac{n_{i}}{N_{i}}
$$

Finally, the 15-environmental states, $E_{S}$, considered for fatigue life calculations extracted from the joint probability distribution of $H_{S}, T_{Z}$, and $U_{10}$ in the area of Blyth [25] are shown in Table 2.

2.2 Sensitivity Analysis. In this work, a sensitivity scheme, combining three sensitivity analyses, i.e., two-dimensional (2D) scatter plots, EE, and Sobol' indices, was developed to establish the least and most influential model input parameters (see Fig. 4). The 2D scatter plot displays the model response in terms of one of their input parameters to observe patterns that reveal a certain degree of correlation between the two variables. In this work, 2D scatter plots were used to visually identify the sensitive factors in the design based on potential shapes or patterns on the plotted data [11]. EE method, in contrast, aims to provide information about: (1) the influence of a certain input parameter in the response and (2) other indirect influences that the parameter may have in the model response due to nonlinear effects and interaction with other input parameters [10]. Scatter plots and EE can be classified as screening methods and are usually used simultaneously due to their efficiency, and simplicity to identify the lessinfluential parameters when complex models with a large number of parameters are analyzed. Nevertheless, they face some shortcomings associated with local sensitivity analysis, which undermine their capacity to identify the most influential factors [10]. 


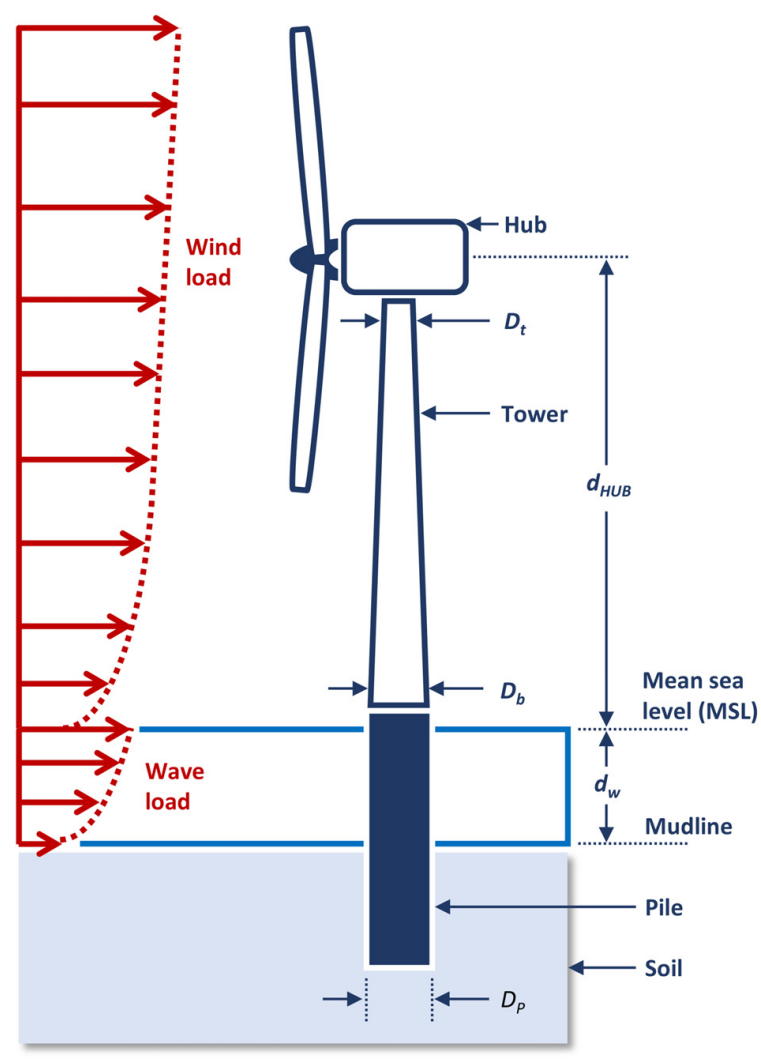

(a)

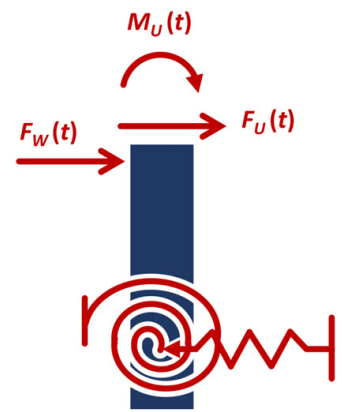

(b)

Fig. 3 Schematic of the OWT loads: (a) turbine and monopile and (b) monopile

Table 1 Main characteristics of the 5 MW OWT

\begin{tabular}{lc}
\hline \hline Parameter & Value \\
\hline Grout thickness & $0.05 \mathrm{~m}$ \\
Transition piece thickness & $0.1 \mathrm{~m}$ \\
Soil Poisson's ratio & 0.2 \\
$\xi$ & $6 \%$ \\
$D_{t}$ & $3.87 \mathrm{~m}$ \\
$D_{b}$ & $6 \mathrm{~m}$ \\
$d_{\text {HUB }}$ & $90 \mathrm{~m}$ \\
$d_{w}$ & $9.3 \mathrm{~m}$ \\
$R_{t}$ & $63 \mathrm{~m}$ \\
$D_{P}$ & $6 \mathrm{~m}$ \\
log(a) & $(11.7-11.3)$ \\
$m$ & $(3-5)$ \\
$t_{p}$ & $67 \mathrm{~mm}$ \\
$k$ & 0.25 \\
SCF & 1.13 \\
Rated speed & $11.4 \mathrm{~m} / \mathrm{s}$ \\
Rotational speed & $12.1 \mathrm{RPM}$ \\
Cut-in speed & $3 \mathrm{~m} / \mathrm{s}$ \\
Cutout speed & $25 \mathrm{~m} / \mathrm{s}$ \\
\hline \hline
\end{tabular}

To overcome that situation, analysis of variance analyses are normally employed, in which the Sobol' approach is one of the most used, and thereby considered as a benchmark for comparing other sensitivity approaches [10]. In this approach, the sensitivity indices are determined based on the variance decomposition of the output samples regarding the contribution of the inputs. There are two main outputs from the Sobol' sensitivity scheme: the firstorder and the total Sobol' indices, respectively. The first-order index accounts for the influence of an individual random variable
(RV) on the total variance of the system, whereas the total Sobol' indices account for the higher-order interactions between all the input parameters [11]. In this study, the information extracted from the total effects is usually used for model reduction alongside the screening methods. The information of the first effects and the variations between the first and total indices, instead, is used for model prioritization, which aims to identify the most influential parameters to draw suggestions of how their uncertainty can be reduced to minimize the variability of the response [11].

Table 2 Environmental states $\left(E_{S}\right)$

\begin{tabular}{lcccc}
\hline \hline State & $H_{S}(\mathrm{~m})$ & $T_{Z}(\mathrm{~s})$ & $U_{10}(\mathrm{~m} / \mathrm{s})$ & $P_{i}(\%)$ \\
\hline 1 & 0.25 & 2.0 & 5.0 & 20.47 \\
2 & 0.25 & 5.2 & 4.9 & 3.73 \\
3 & 0.25 & 4.0 & 11.8 & 21.76 \\
4 & 0.25 & 5.6 & 15.7 & 3.85 \\
5 & 0.25 & 5.8 & 20.6 & 1.00 \\
6 & 0.75 & 3.4 & 6.7 & 8.62 \\
7 & 0.75 & 5.3 & 5.8 & 13.25 \\
8 & 0.75 & 5.5 & 11.7 & 5.58 \\
9 & 1.25 & 5.2 & 8.8 & 10.66 \\
10 & 1.25 & 8.0 & 8.5 & 1.25 \\
11 & 1.75 & 6.0 & 9.9 & 4.83 \\
12 & 1.75 & 6.7 & 16.2 & 0.55 \\
13 & 2.4 & 6.8 & 12.8 & 3.54 \\
14 & 3.4 & 7.8 & 14.5 & 0.77 \\
15 & 3.3 & 9.7 & 18.7 & 0.14 \\
\hline \hline
\end{tabular}




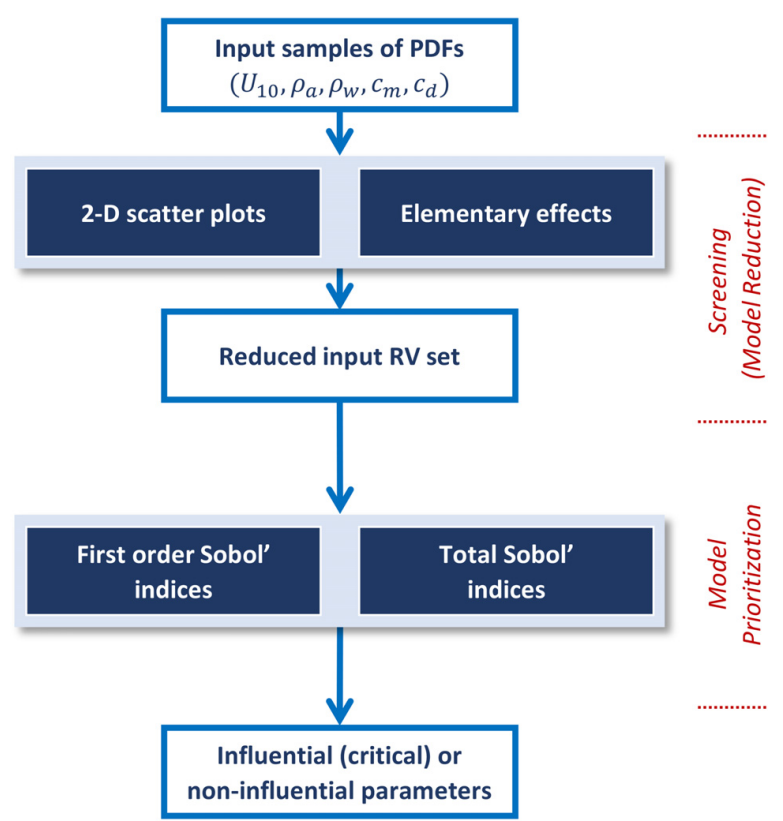

Fig. 4 Schematic representation of the sensitivity analysis scheme for the OWT system

2.3 Uncertainty Quantification. The Uncertainty quantification scheme presented in this work is divided into three steps: probabilistic modeling, uncertainty propagation, and statistical certification (see Fig. 5). In the probabilistic modeling, a consistent probabilistic law is specified for the joint PDFs of the model parameters using the known information about them. Then, the uncertainty propagation step uses Monte Carlo simulation to access how this input PDF is modified by the model operator. Finally, the statistical certification step is devoted to the visualization of the results, in terms of their PDFs, and probability of failure, $P_{f}$.

2.3.1 Probabilistic Modeling. The probabilistic modeling step in the UQ scheme is schematically represented in Fig. 6, where the selected RVs (see Table 3) from the sensitivity analysis were classified in accordance to the type of information available: (1) physics-informed theoretical information and (2) data-driven information. Physics-informed theoretical information, on the one hand, is considered to ensure the consistency of the probability distributions used in the stochastic model, as it forces them to

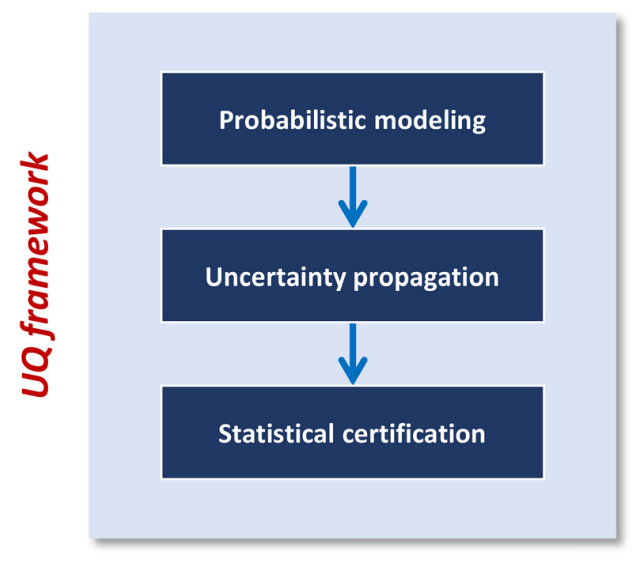

Fig. 5 Schematic representation of the uncertainty quantification framework respect the laws of physics and their underlying constraints, e.g., support positivity, finite variance due to limited fluctuations, and smooth decaying in the tails. This information influences the form of the probability distribution to be chosen, as well as the choice of acceptable support, ignoring these restrictions can lead to a probabilistic model that violates the fundamental physics of the problem. Data-driven information, on the other hand, the latter information, obtained from experiments or field measurements datasets, guides the choice of plausible values for the hyperparameters of probabilistic distributions, as well as their statistics.

Thus, joint PDF of the model input needs to be specified, mandatorily, with the aid of these two types of information, since, by neglecting the restrictions arising from physics, the probabilistic model will very likely be inconsistent, while when not considering reliable data, the nominal values of the hyperparameters and statistics can be very flawed so that the predictions are very different from the reality.

In a scenario where a large dataset with information about the model parameters, and their mutual dependence, is available, classical inference techniques of nonparametric statistics (e.g., kernel density methods) may be used to construct the probabilistic model. However, when a small (i.e., sparse) set of observations is available or, in the limit, only theoretical information is given, this classical approach is unfeasible; thus, another strategy is necessary for robust inference. In this second context, the most consistent approach is to use an information-theoretic approach based on the maximum entropy principle (MaxEnt) which seeks to specify as the model input joint-distribution the maximum entropy probabilistic law that is compatible with the known physicsinformed or data-driven-information about the system parameters $[31,32]$. The idea behind this formalism is that the obtained probabilistic distribution is consistent because it is compatible with the constraints imposed by the physics and the known statistical information, as well as conservative since it is the most uncertain

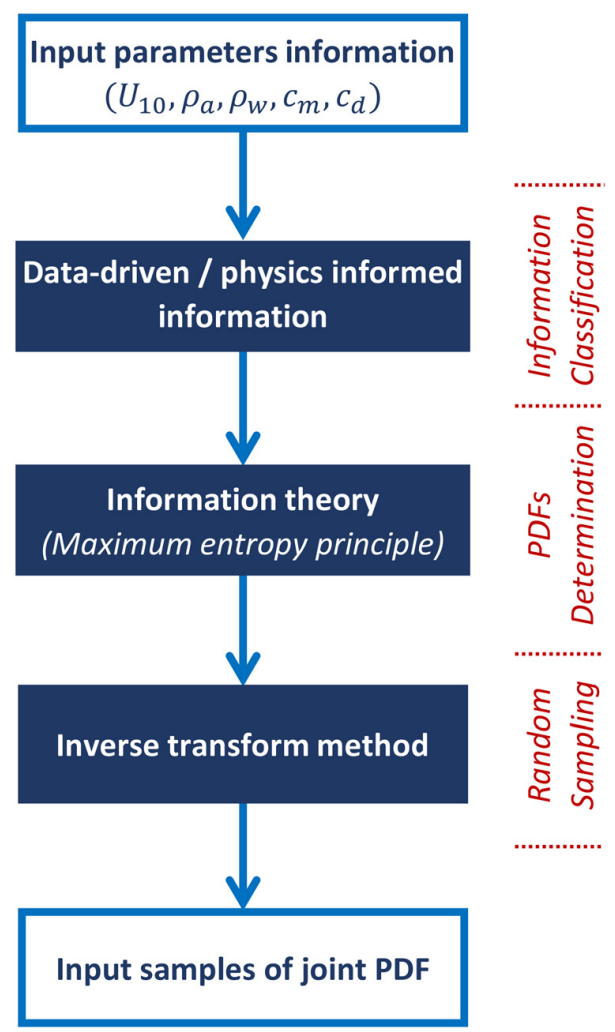

Fig. 6 Schematic representation of the probabilistic modeling scheme 
Table 3 Information about the random variables

\begin{tabular}{|c|c|c|c|c|}
\hline $\mathrm{RV}$ & Data-driven & Physics-informed & MaxEnt & References \\
\hline$\rho_{\text {air }}$ & $\begin{array}{l}\text { Supp }=[1.146,1.315] \\
\mathrm{E}[X]=1.27 \\
\delta_{X}=3[1-4] \%\end{array}$ & $\begin{array}{l}\text { Supp }=\mathbb{R}^{+} \\
\mathrm{E}[X]=m, 0<m<+\infty \\
\mathrm{E}\left[X^{-2}\right]=c, c<+\infty \\
\mathrm{E}[\log (\mathrm{X})]=d, d<+\infty\end{array}$ & Gamma & Theoretical $[7,22,27]$ \\
\hline$\alpha$ & $\begin{array}{l}\text { Supp }=[0.1,0.2] \\
\mathrm{E}[X]=0.14 \\
\delta_{X}=3[0-5] \%\end{array}$ & $\begin{array}{l}\mathrm{Supp}=\mathbb{R}^{+} \\
\mathrm{E}[X]=m, 0<m<+\infty \\
\mathrm{E}\left[X^{-2}\right]=c, c<+\infty \\
\mathrm{E}[\log (X)]=d, d<+\infty\end{array}$ & Gamma & Theoretical $[7,21,28]$ \\
\hline$U_{10}$ & $\begin{array}{l}\text { Supp }=[0,63] \\
\mathrm{E}[X]=U_{10}(\text { state }) \\
\mathrm{E}\left[(X-\mu)^{2}\right]=\left(\sigma_{\mathrm{NTM}}\right)^{2}\end{array}$ & $\begin{array}{l}\text { Supp }=\mathbb{R}^{+} \\
\mathrm{E}\left[X^{-2}\right]=c, c<+\infty \\
\mathrm{E}[\log (X)]=d, d<+\infty \\
\mathrm{E}\left[(\log (X)-\mu)^{2}\right]=m, \\
0<m<+\infty\end{array}$ & Log-normal & Data [25] \\
\hline$\rho_{\mathrm{sw}}$ & $\begin{array}{l}\text { Supp }=[1,020,1,030] \\
\mathrm{E}[X]=1,027 \\
\delta_{X}=1[0-2] \%\end{array}$ & $\begin{array}{l}\text { Supp }=\mathbb{R}^{+} \\
\mathrm{E}[X]=m, 0<m<+\infty \\
\mathrm{E}\left[X^{-2}\right]=c, c<+\infty \\
\mathrm{E}[\log (\mathrm{X})]=d, d<+\infty\end{array}$ & Gamma & Theoretical $[22,28]$ \\
\hline$C_{m}$ & $\begin{array}{l}\text { Supp }=[1.7,2.15] \\
\mathrm{E}[X]=2 \\
\delta_{X}=10[6-30] \%\end{array}$ & $\operatorname{Supp}=[a, b]$ & Uniform & Theoretical [21,22] \\
\hline$C_{d}$ & $\begin{array}{l}\text { Supp }=[0.7-1.2] \\
\mathrm{E}[X]=0.95 \\
\delta_{X}=10[6-25] \%\end{array}$ & $\begin{array}{l}\operatorname{Supp}=[a, b] \\
\mathrm{E}[\log (X-a)]=d \\
\mathrm{E}[\log (b-X)]=e|d| \text { and }|e|<+\infty\end{array}$ & Generalized Beta & Theoretical [21,22] \\
\hline$H_{S}$ & $\begin{array}{l}\mathrm{E}[X]=\text { lumped } \\
\delta_{X}=0.10[6.5-16] \%\end{array}$ & $\begin{array}{l}\text { Supp }=\mathbb{R}^{+} \\
\mathrm{E}[X]=m, 0<m<+\infty \\
\mathrm{E}\left[X^{-2}\right]=c, c<+\infty \\
\mathrm{E}[\log (X)]=d, d<+\infty\end{array}$ & Gamma & Data [25] \\
\hline$T_{Z}$ & $\begin{array}{l}\mathrm{E}[X]=\text { lumped } \\
\delta_{X}=0.10[3-16] \%\end{array}$ & $\begin{array}{l}\text { Supp }=\mathbb{R}^{+} \\
\mathrm{E}[X]=m, 0<m<+\infty \\
\mathrm{E}\left[X^{-2}\right]=c, c<+\infty \\
\mathrm{E}[\log (X)]=d, d<+\infty\end{array}$ & Gamma & Data [25] \\
\hline $\mathrm{E}$ & $\begin{array}{l}\text { Supp }=[189,230] \\
\mathrm{E}[X]=208 \\
\delta_{X}=5.4 \% \\
\mathrm{E}\left[((X-\mu) / \sigma)^{3}\right]=0.16 \\
\mathrm{E}\left[((X-\mu) / \sigma)^{4}\right]=2.68\end{array}$ & $\begin{array}{l}\mathrm{Supp}=\mathbb{R}^{+} \\
\mathrm{E}[X]=m, 0<m<+\infty \\
\mathrm{E}\left[X^{-2}\right]=c, c<+\infty \\
\mathrm{E}[\log (X)]=d, d<+\infty\end{array}$ & Gamma & Data [30] \\
\hline$\rho_{\mathrm{ST}}$ & $\begin{array}{l}\mathrm{E}[X]=8500 \\
\delta_{X}=1[0-2] \%\end{array}$ & $\begin{array}{l}\text { Supp }=\mathbb{R}^{+} \\
\mathrm{E}[X]=m, 0<m<+\infty \\
\mathrm{E}\left[X^{-2}\right]=c, c<+\infty \\
\mathrm{E}[\log (X)]=d, d<+\infty\end{array}$ & Gamma & Theoretical [17] \\
\hline$S_{N}$ & $\begin{array}{l}\text { Supp }=[0,1] \\
\mathrm{E}[X]=0.1 \\
\delta_{X}=10[5-15] \%\end{array}$ & $\begin{array}{l}\operatorname{Supp}=[0,1] \\
\mathrm{E}[\log (\mathrm{X})]=d \\
\mathrm{E}[\log (1-X)]=e \\
|d| \text { and }|e|<+\infty\end{array}$ & Beta & Theoretical [26] \\
\hline$E_{\mathrm{SO}}$ & $\begin{array}{l}\text { Supp }=[4000,7500] \\
\mathrm{E}[X]=5160 \\
\delta_{X}=30[20-50] \%\end{array}$ & Supp $=[a, b]$ & Uniform & Theoretical and expert [30] \\
\hline
\end{tabular}

distribution (thus, covering a bigger portion of the sample space). To the best of the authors' knowledge, a distribution obtained in this way is the most consistent one in a scenario with sparsely known statistical information or even when only theoretical information is available [32].

As far as correlated random variables are concerned, the most conservative approach is to assume them independent random variables, if there is no statistical information about the crosscorrelation of the parameters is available. The latter follows directly from MaxEnt formalism since assuming values of crosscorrelation would add bias to the analysis [31,32].

After the information concerning the selected RVs was sorted, the joint PDF of the input RVs was determined either using maximum MaxEnt or the kernel density estimator when a large dataset was available [16,34]. Finally, the inverse transform method was used to randomly draw samples from the input joint PDF, which later was employed in the processing step [16].

2.3.2 Uncertainty Propagation. The uncertainty propagation scheme used in the proposed model is illustrated in Fig. 7. The uncertainty from the input parameters was propagated into the model to obtain samples of the model response parameter, i.e., $D_{30}$, which later will be used to infer its statistical information (e.g., mean, standard deviation, and PDF). As it was discussed in Ref. [34], it is necessary to run several millions of scenarios, also known as MC simulation to obtain meaningful results from an OWT probabilistic analysis for ten RVs. Thus, in this study, an efficient surrogate was constructed to conduct the processing step due to the computational cost involved in the running of the complex deterministic model introduced previously. 
Among the different surrogates available in the literature, the kriging approach was shown to produce more accurate results for the fatigue life estimations [35]. Hence, in this study, a generalized kriging metamodel with a linear trend and Mantern 3/2 correlation function was selected to replace the deterministic model shown in Fig. 2. To ensure accurate approximations of the surrogate, a dataset of 2250 points, a combination of 150 samples per environmental condition, was drawn from the deterministic model using the Latin hypercube sampling (LHS) approach, wherein the UQlab toolbox, free software for UQ in MATLAB, was used to obtain the LHS sample and kriging model [36]. In addition, to further improve the capabilities of the kriging surrogate, a natural log transformation was applied to the model response to improve the surrogate interpolation process by having a more sparse resolution of the results.

After the surrogate was developed, the MC approach was utilized to generate more than $8 \times 10^{8}$ samples from the $D_{30}$, to determine their statistical information. Lastly, to show the accuracy of estimators determined from the samples generated from $\mathrm{MC}$, the mean square convergence criterion was conducted to ensure the convergence of the results for the selected number of samples in this study [33].

2.3.3 Statistical Certification. In this stage, the samples obtained during processing were employed to (1) construct the PDF and cumulative density function (CDF) for the fatigue damage of the structure, and (2) to compute the structural probability of failure $\left(P_{f}\right)$ during an operation life of at least 30 years.

To verify the accuracy of the proposed UQ framework, the Kolmogorov-Smirnov (KS) goodness of fit test was carried out The KS test aims to prove the hypothesis that the chosen probabilistic model, i.e., CDF, is a suitable representation of the physical behavior of the parameter of interest, with a certain significance level, $\alpha_{\mathrm{KS}^{-}}$that is, $0.1,1$, or $5 \%$. In this study, $0.1 \%$ was used since it represents the better fit [29].

Regarding the structural reliability, this is typically represented by the $P_{f}$, which can also be defined as $1-R_{\mathrm{OWT}}$, where $R_{\mathrm{OWT}}$ is the reliability of the structure in percentage units. To determine the $P_{f}$, the limit state function $Z$ was defined in terms of the fatigue limit state (FLS) design criterion, which is 1 for this study, and the calculated damage of the response for a service life of 30 years, $D_{30}$

$$
Z=\mathrm{FLS}-F_{D 30}(v)
$$

Then, the $P_{f}$ was calculated as the probability of $\mathrm{Z}$ being less or equal to zero, which was found by sampling $N_{P}$ samples from the distribution of $D_{30}$. Finally, the error relative to the true target

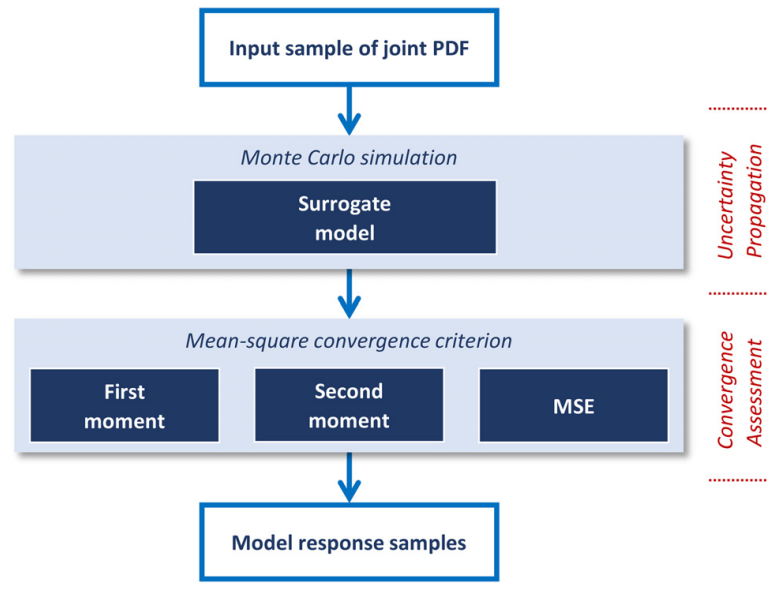

Fig. 7 Schematic representation of the propagation of uncertainties step probability of failure, $P_{f}^{T}$, associated with the selected $N_{P}$, as well as the variability of the $P_{f}$ was given in terms of their relative error, $\varepsilon_{p f}$, and coefficient of variation, $\delta_{p f}$, respectively [29],

$$
\begin{gathered}
\varepsilon_{P f}=(200) \sqrt{\frac{\left(1-P_{f}^{T}\right)}{\left(N_{p} * P_{f}^{T}\right)}} \quad[\%] \\
\delta_{P f}=\left(\frac{100}{P_{f}}\right) \sqrt{\frac{\left(1-P_{f}\right) P_{f}}{N_{p}}} \quad[\%]
\end{gathered}
$$

2.4 Specific Analyses. The specific analyses for specific aim 1 and 2 are introduced in the Secs. 2.4.1 and 2.4.2, respectively.

2.4.1 Model Response Parameters That Impact Fatigue Life. To quantitatively assess the influence of input parameters in the design of OWT in terms of total damage accumulated on the structure for 30 years, $D_{30}$, the overall analysis was divided as follows: model reduction, and model prioritization. On the one hand, in the model reduction analysis 12 input-model parameters were selected, e.g., $T_{Z}, U_{10}, H_{S}, \rho_{\text {air }}, \alpha, c_{d}, S_{N}$, to be investigated to identify the less influential parameters and potentially fixed them - that is, transforming into deterministic quantities. The analysis was conducted using the efficient $\mathrm{EE}$ approach for four different environmental conditions (1) cut-in speed to rated-speed, (2) rated-speed, (3) rated-speed and cutout speed, and (4) extreme conditions (highest speed interval recorded of the environmental states). Furthermore, the 2D scatter plots of the variables were also computed to verify the results. In the model prioritization, on the other hand, part of the parameters that significantly contribute to the total variance was assessed by conducting the first and total Sobol' global-sensitivity approach. Then, recommendations to improve the model robustness were provided based on reducing epistemic uncertainty of influential parameters.

2.4.2 Probability Distributions of the Model Response Parameters. The PDFs for the model input parameters were established following the procedure in section 2.3.1 and using the datadriven and physics-informed information displayed in Table 3. For example, the PDFs for $\rho_{\text {air }}, \alpha, c_{d}, S_{N}$ was derived using datadriven information about the limits, the sample mean, $\mathrm{E}[X]$, and coefficient of variation, $\delta_{X}$, as well as physical-informed information regarding their domain, support, finite variance, $\mathrm{E}[\mathrm{X}-2]=c$ for $c<\infty$, and tail behavior, $\mathrm{E}[\log (\mathrm{X})]=d$ for $d<\infty$, in which $d<\infty$ represents a decaying right tail converging to zero at d. This information about the RVs is available in the literature, such as books and standards. The PDFs of $E_{\mathrm{so}}$, and $c_{m}$, instead, were derived based on information about their sample mean and limits only. From the aforementioned distributions, random samples were drawn and propagated into the deterministic model to generate samples of $D_{30}$, i.e., synthetic data, with MC simulation; and then, an informational maximum entropy distribution was constructed to model the variability of synthetic data. Finally, the consistency of the synthetic data was verified using the mean square convergence criterion, whereas the suitability of the nonparametric fit is analyzed using the KS goodness of fit test with an $\alpha_{\mathrm{KS}}$ of $0.1 \%$.

\section{Results and Discussions}

In this section, the results obtained from the analyses for specific aims 1 and 2 are presented and discussed.

To begin with, Fig. 8 and Table 4 depict the validation results of the developed generalized kriging metamodel for the environmental states $1,8,11$, and 15 described in Table 2. From the statistics, it stands out the high levels of correlation between the surrogate model and the computational model, as well as low error values, which in turn show the high level of accuracy of the predicted values by the developed surrogate model. For example, the 


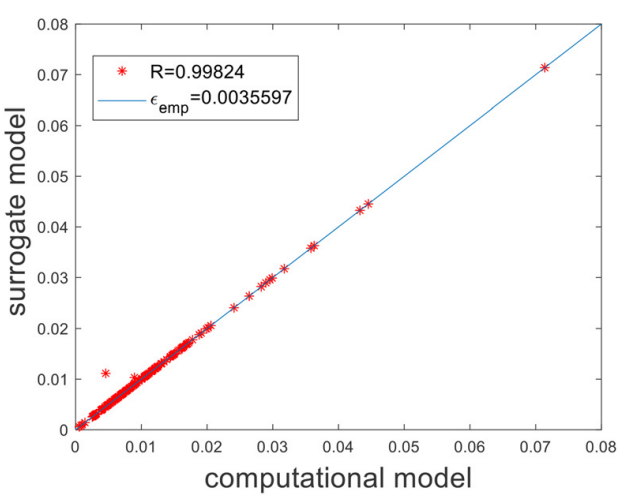

(a)

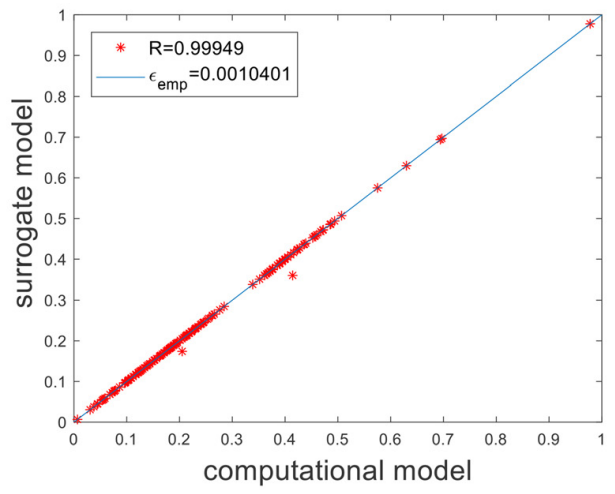

(c)

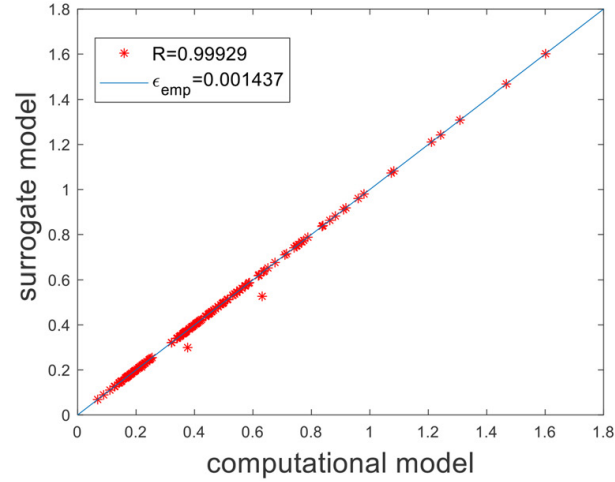

(b)

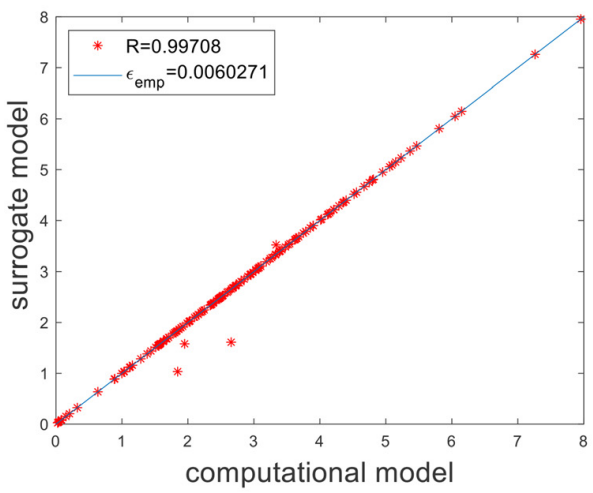

(d)

Fig. 8 Surrogate modeling validation: (a) environmental state \#1; (b) environmental state \#8; (c) environmental state \#11; and (d) environmental state \#15

training process-wherein the accuracy is presented in terms of the root-mean-square error (RMSE), and the leave-one-out error, $\varepsilon_{\mathrm{LOO}}$ - showed error values below $2 \times 10^{-3}$ for the RMSE, and 0.15 for the $\varepsilon_{\text {LOO }}$. Similarly, the validation process-where the accuracy is assessed in terms of the correlation coefficient, $R$, and the empirical error, $\varepsilon_{\mathrm{emp}}$ - showed $R$ values between the validation set and the surrogate prediction in the order of $99 \%$, and $\varepsilon_{\mathrm{emp}}$ values below $1 \times 10^{-3}$ for all the environmental states used in this analysis. As a result, the ratio of 10 samples per random variable selected for the analysis - which resulted in a dataset of 150 samples per environmental state, and thereby total dataset of 2250 samples - to train the Kriging surrogate model provided a fairly accurate approximation of the response of the deterministic model.

The rest of the results of the paper: (1) sensitivity analysis of the model input parameters, (2) calculation of the probability distributions of model input parameters, and (3) analysis of the impact of the proposed UQ scheme on the OWT structural reliability is discussed in Secs. 3.1 through 3.3.

3.1 Sensitivity Analysis of the Model Input Parameters. The results of the influence of input parameters in the design of OWT using the sensitivity scheme for environmental states $\left(E_{S}\right) 6$, 8,11 , and 15 , which also represent four different loading conditions, are depicted in Figs. 9-11.

As can be seen from the graphs, in particular Fig. 11, it is clear that $U_{10}$ presented the higher sensitivities, followed by the air density, $\rho_{\text {air }}$, particularly for the first three loading conditions, whereas $H_{S}$ had the third-largest contribution in the third and fourth conditions. The impact of $U_{10}$ on the variance of the response can be explained by the fact that this parameter is directly proportional to wind pressure, which increases quadratically with the wind speed. As a result, small variations in $U_{10}$ can significantly increase the thrust force and the mudline bending moment of the structure, which in turn, increases the accumulated structural damage during the OWT operational life.

Parameters $\alpha$ and $\rho_{\text {air }}$ also presented slight contributions to the response in particular $\rho_{\text {air. }}$. Although $\alpha$ and $\rho_{\text {air }}$ also impact the wind loads, albeit, to a lesser degree than $U_{10}$, it is normal to assume these parameters as constant values in OWT design standards $[21,22]$. However, recent studies, have shown that $\alpha$ and $\rho_{\text {air }}$ may vary considerably from the values suggested by design standards, thus impacting the wind loads $[7,37]$. The air density $\left(\rho_{\text {air }}\right)$, on the one hand, is typically determined as the expected value of the air density based on the long-term site environmental conditions - which are humidity, temperature, and pressure-and shows a low variability, i.e., $\delta_{X}$ ranging between 1 and $4 \%$ in most cases. Nevertheless, studies have shown some locations that present higher values [37]. This is a problematic fact, because changes in $\rho_{\text {air }}$ may produce around $15 \%$ variability in long-term average power production, and $26 \%$ in hourly power production. The wind and wave loading also change since $\rho_{\text {air }}$ is directly proportional to the wind pressure $[37,38]$. On the other hand, the parameter $\alpha$ is considered, for design purposes, an average value based on longterm conditions. In other words, the expected value of the shear

Table 4 Surrogate model validation metrics

\begin{tabular}{lcccc}
\hline \hline State & RMSE & $\varepsilon_{\text {LOO }}$ & $R$ & $\varepsilon_{\text {emp }}$ \\
\hline 1 & $1.23 \times 10^{-10}$ & 0.106 & 0.99 & $3.5 \times 10^{-3}$ \\
8 & $1.23 \times 10^{-10}$ & 0.106 & 0.99 & $1.4 \times 10^{-3}$ \\
11 & $1.23 \times 10^{-10}$ & 0.106 & 0.99 & $1 \times 10^{-3}$ \\
15 & $1.23 \times 10^{-10}$ & 0.107 & 0.99 & $6 \times 10^{-3}$ \\
\hline \hline
\end{tabular}


coefficient in terms of the long-term mean wind speed at different altitudes is recorded for a specific location. Since the wind speed has daily and seasonal variations, $\alpha$ also varies. For example, it was observed that the mean value of $\alpha$ can oscillate between 0.08 and 0.15 in coastal and offshore areas, and up to 0.35 in onshore areas [29]. Regarding the coefficient of variation of the parameter $\alpha, \delta_{\alpha}$, it has been reported that high wind speeds present small $\delta_{\alpha}$ values, around than $10 \%$, whereas small wind speeds present large $\delta_{\alpha}$ values, more than $50 \%$ [7]. This in turn impacts the wind speed at the hub height.

Whilst $U_{10}$ was the input parameter of the wind load that consistently presented the higher index for each condition, the inputs parameters for the wave load, i.e., $c_{d}, c_{m} T_{Z}$, and especially $H_{S}$, showed a slight increase in the third and fourth loading condition in comparison with the previous ones. This can be explained due to the action of the pitch control, which reduces the influence of
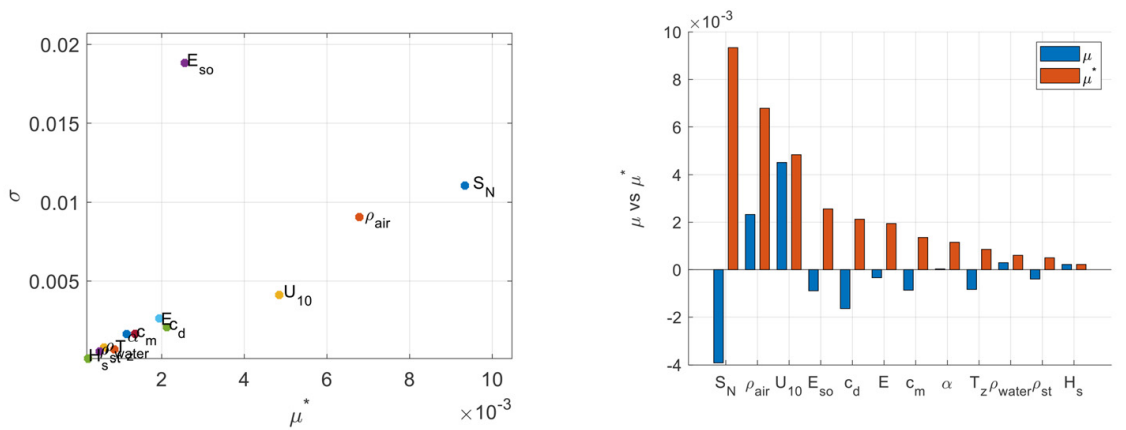

(a)
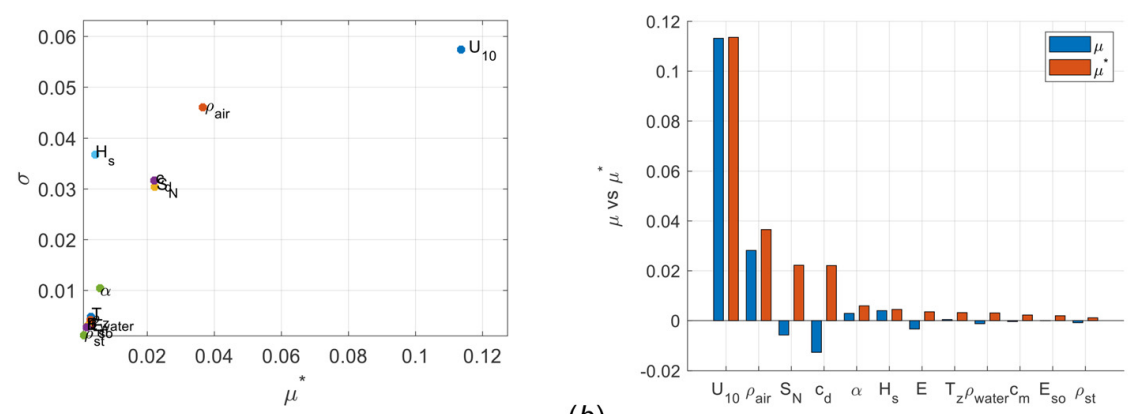

(b)
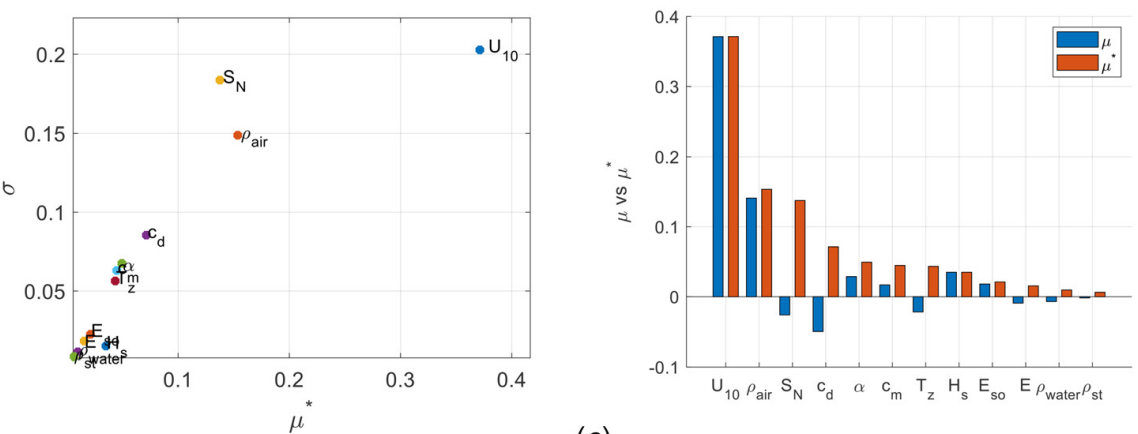

(c)
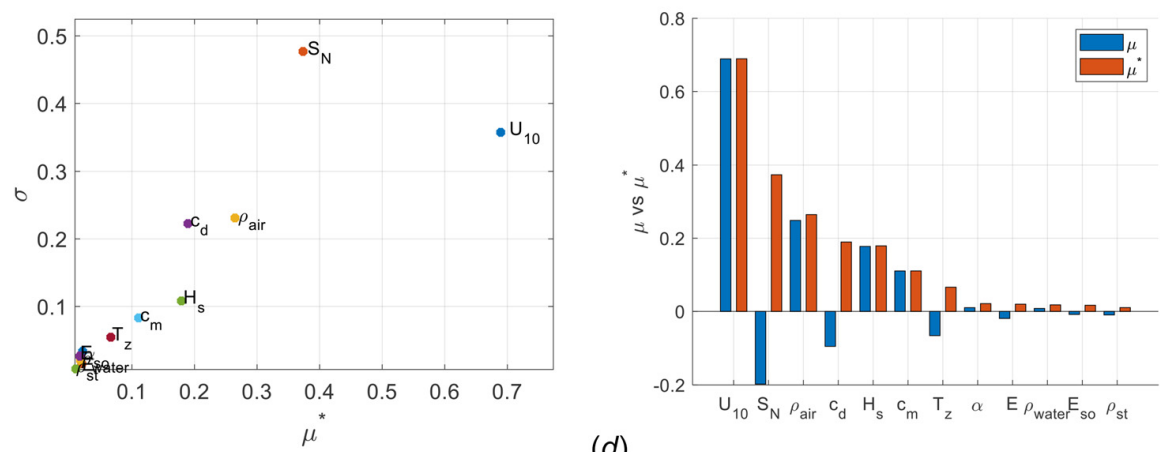

(d)

Fig. 9 Elementary effects sensitivity plots: (a) environmental state \#6, (b) environmental state \#8, (c) environmental state \#11, and (d) environmental state \#15 

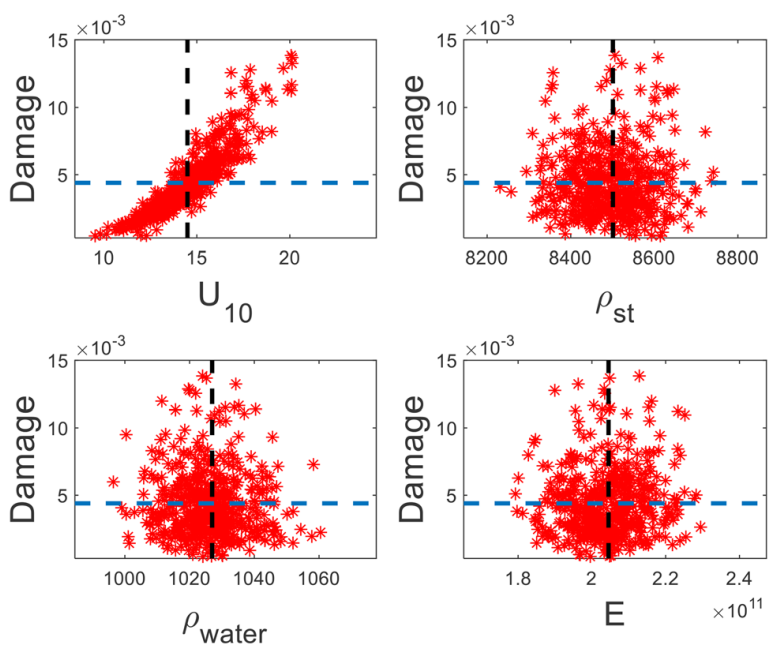

Fig. 10 Scatter plots for sensitivity analysis environmental state \# 11 (rated power conditions)

the wind load, thereby giving more importance to the wave loading in the fatigue calculations.

Regarding the remaining $\mathrm{RVs}$, such as $E, S_{N}, \rho_{\mathrm{st}}$, as well as $\rho_{\text {water }}$, they only had a marginal contribution to the variance of the response. For example, the variability of $\mathrm{E}, \rho_{\mathrm{st}}$, and $S_{N}$ arises from the material properties and welding quality of the tubular section of the pile. The variability of $\rho_{\text {water }}$ is mainly due to measuring errors and variability of other parameters such as humidity, temperature, and salinity of the seawater employed to compute $\rho_{\text {water }}$.
Although the aforementioned parameters may have a mild impact on the wind and wave loading, the overall contribution to the damage in terms of direct effects and nonlinear effects was not significant in this study in comparison with other model input parameters.

In summary, considering that all model input parameters for this analysis do not behave equally at different environmental states, the selection of the most and less important input parameters should not only include the information from all the operation conditions, but also about the potential higher-order and nonlinear interactions with other parameters [11]. Regarding the less influential parameters $\left(E, \rho_{\text {st }}\right.$, and $\left.\rho_{\text {water }}\right)$ they consistently presented negligible effects in terms of the direct effects to the response, i.e., $u^{*}$ index in the EE method and first-order Sobol' index. These parameters showed negligible nonlinear interactions with other input parameters based on the $\sigma$ index of the EE approach and total Sobol' indices. As a result of that, their scatter plots in Fig. 10 do not show a clear pattern that implies correlations with the structural damage, and the input parameters $E, \rho_{\text {st }}$, as well as $\rho_{\text {water }}$, can be transformed to deterministic quantities to improve efficiency and reduce the complexity of the problem since their contribution was proved to be negligible in all the analyses.

The most influential parameters, in contrast, were found to be $U_{10}$ and $\rho_{\text {air }}$, so to reduce the variance of the response, it is recommended to collect more data and conduct more sophisticated simulations to reduce the uncertainty of those parameters. Finally, other environmental parameters, such as $E_{\mathrm{so}}, T_{Z}, H_{S}$, and $\alpha$, are not recommended to be discarded since they may significantly vary for other locations not considered in this study. Consequently, ignoring their variability may cause significant impacts on the fatigue damage, particularly large OWT that is more sensitive to dynamic amplification due to wave loading [34,39].

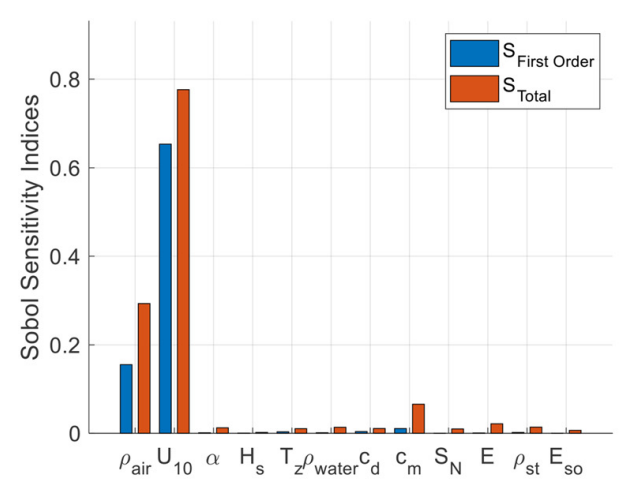

(a)

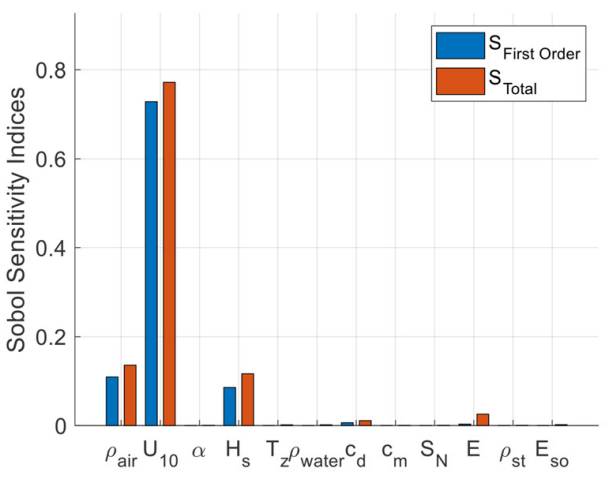

(c)

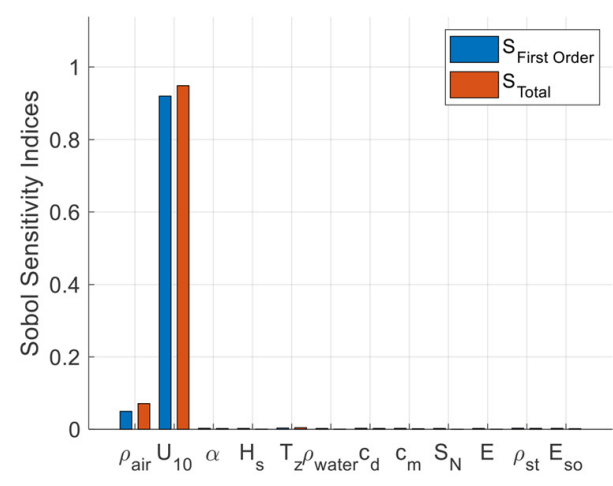

(b)

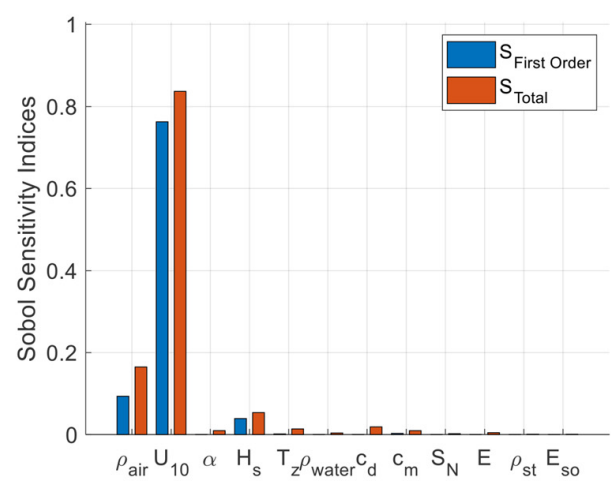

$(d)$

Fig. 11 Sobol' sensitivity analysis: (a) environmental state \#6, (b) environmental state \#8, (c) environmental state \#11, and (d) environmental state \#15 


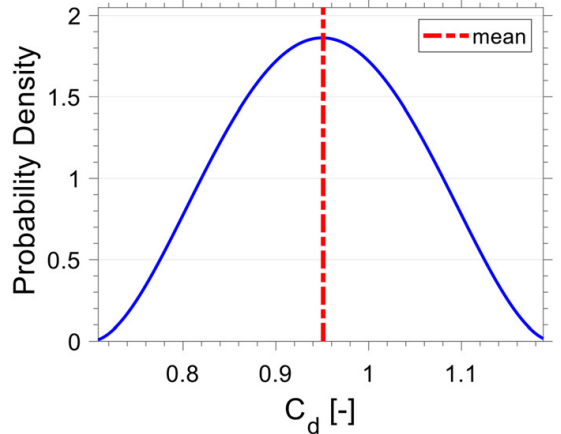

(a)

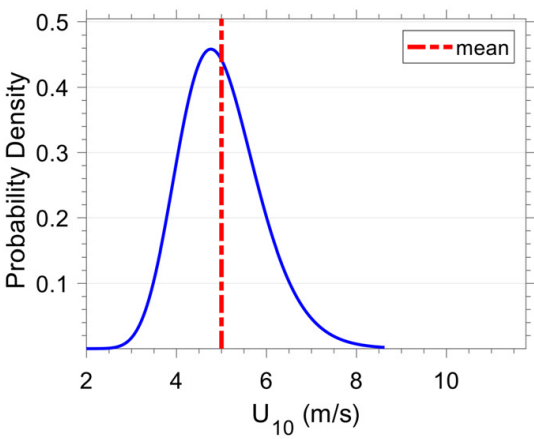

(c)

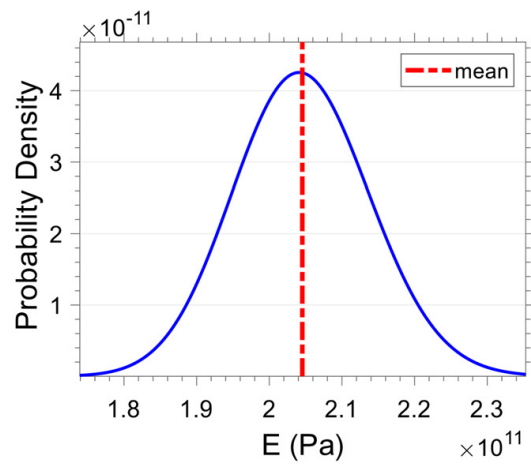

(e)

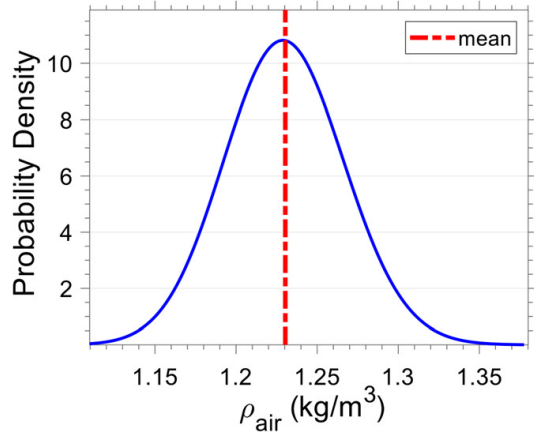

(b)

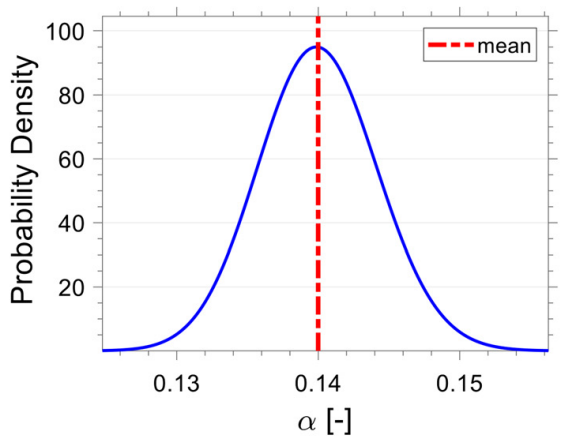

(d)

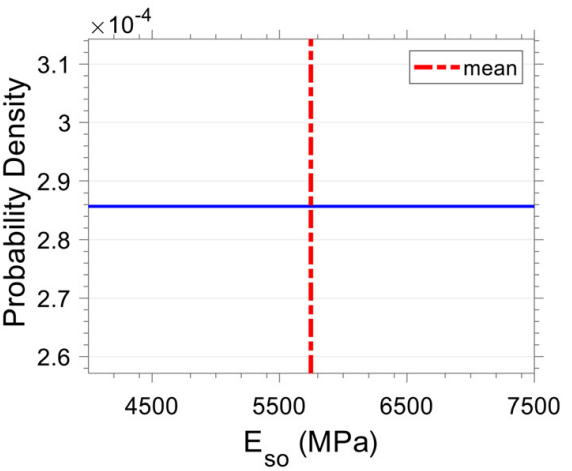

$(f)$

Fig. 12 MaxEnt probability densities of input random variables: (a) inertia coefficient, $(b)$ air density, $(c)$ 10-min wind speed, $(d)$ shear coefficient, $(e)$ modulus of elasticity, and $(f)$ soil modulus of elasticity

3.2 Probability Distributions of the Model Input Parameters. In this section, the model input PDFs determined using the systematic UQ scheme for the Blyth location, as well as the risks of using arbitrary distributions versus the UQ scheme are presented and discussed. To begin with, Fig. 12 shows the maximum entropy marginal PDFs, for $6 \mathrm{RV}$ (i.e., $c_{d}, \rho_{\text {air }}, U_{10}, \alpha, E$, and $E_{\mathrm{SO}}$ ) of the total $12 \mathrm{RVs}$ used for this study. Their PDFs were constructed using MaxEnt for a different set of constraints based on available data-driven and physics-informed information about the selected RVs and the model response (see Table 3) [31,32].

Figure 12(a) depicts the PDF for the inertia coefficient, $c_{d}$, which was constructed considering three constraints that characterize the physics of this structural parameter: (1) the domain of $c_{d}$ is a strictly positive finite interval in the range of $0.7-1.2$, (2) the expected value of $c_{d}$ must be the same that its mean value $\left(\mathrm{E}\left[c_{d}\right]=\mu_{\mathrm{cd}}\right)$, and (3) the geometric means of $c_{d}$ centered at the support limits must be finite $\left(\mathrm{E}\left[\log \left(c_{t}-a\right)\right]=<\infty\right.$ and $\left.\mathrm{E}\left[\log \left(b-c_{t}\right)\right]=<\infty\right)$. From the set of constraints imposed on the problem, the result of the application of the MaxEnt yields the generalized beta distribution $[31,32]$. Structural parameters such as the elastic modulus, $E$, and the steel density, $\rho_{\text {st }}$, and also to environmental parameters like, $\rho_{\text {water }}$ and $\rho_{\text {air }}$, and shear coefficient $\alpha$, were also subjected to the same set of constraints except for $E$ and $\rho_{\text {st }}$ whose domain is the set of positive real numbers, $\mathbb{R}^{+}$. Hence, the Gamma PDF was utilized for the aforementioned parameters, in which their PDFs can be seen in Figs. 12(a), 12(d), and 12(e). The 10-min wind instantaneous speed, $U_{10}$, MaxEnt distribution (Fig. 12(c)) was found to be the lognormal distribution, whereas the significant wave height, $H_{S}$, and the up-crossing wave period, $T_{Z}$, were modeled as a gamma random variable.

The soil stiffness, $E_{\mathrm{so}}$, MaxEnt density, in contrast, was found to be the uniform distribution. This is because the estimation of soil parameters usually present high uncertainty $\left(\delta_{X}\right.$ ranging from 30 to $50 \%$ ) in the absence of reliable information provided by geotechnical surveys or experiments [6]. The parameter $c_{m}$ was also modeled as uniform distributions since only information 


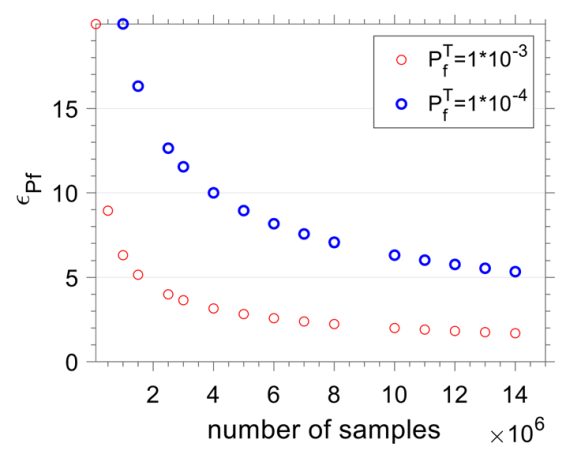

(a)

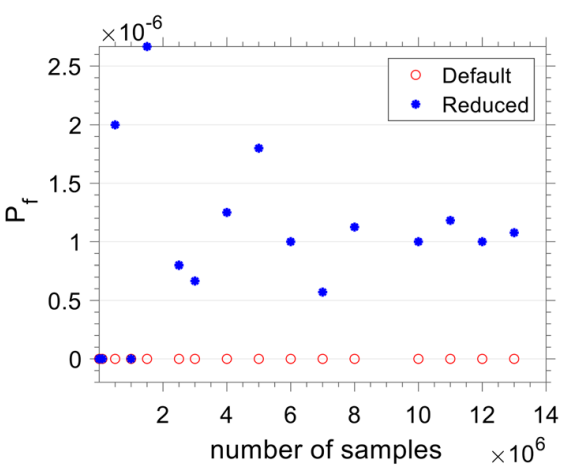

(b)

Fig. 13 Convergence metrics: (a) convergence of relative error for two target probability of failures used in industry and $(b)$ convergence of probability of failure for the reduced and default case

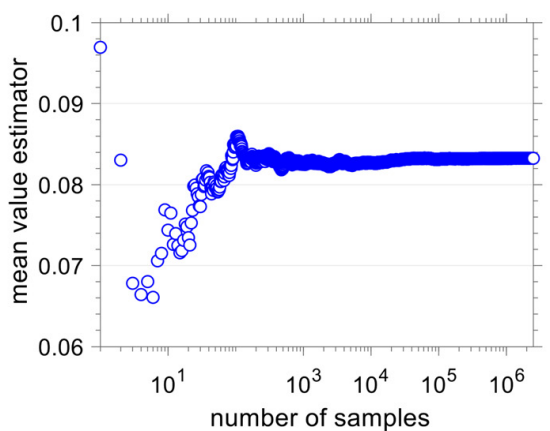

(a)

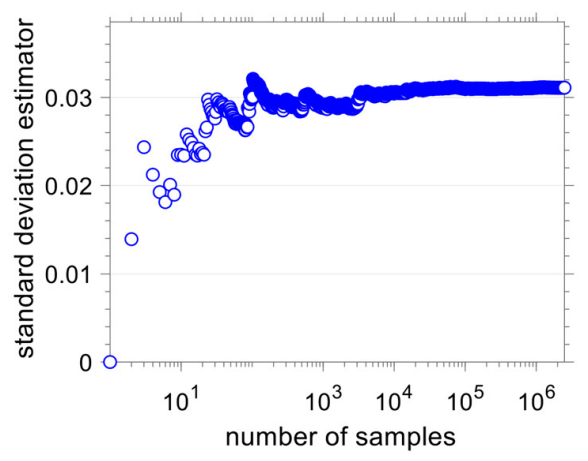

(b)

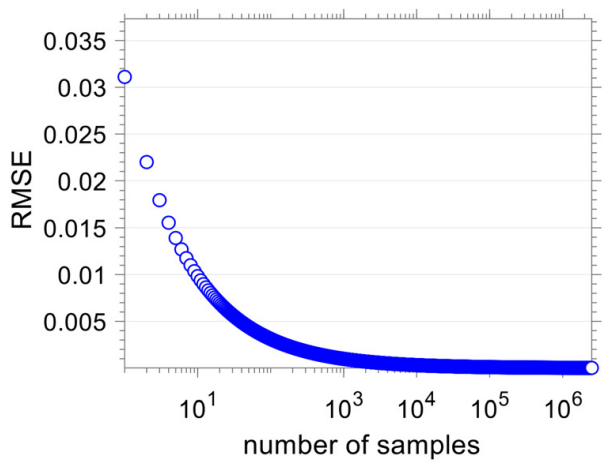

(c)

Fig. 14 MC convergence metrics: (a) mean, (b) STD, and (c) RMSE about their ranges was available [28]. The hyperparameters and other statistics of the aforementioned probabilistic models used in this study, i.e., Gamma, beta, and log-normal, were found from literature as indicated in Table 3.

Since the presented UQ scheme consistently models the randomness in input parameters based on the maximization of the uncertainty in terms of the type of available information, the need to make additional assumptions to determine the input distributions can be minimized. Therefore, considerably reducing the bias that can be potentially passed on to the next steps in the probabilistic modeling, i.e., processing and postprocessing, and ultimately providing more reliable approximations of the response [33].

3.3 Impact of the Uncertainty Quantification Scheme on the Offshore Wind Turbine Structural Reliability. In this section, the results concerning (1) the effects on the reliability estimation due to inconsistencies in its calculation and (2) the impacts of the UQ scheme of the most sensitive input parameters on the structural damage of the OWT are presented and discussed.

3.3.1 Structural Reliability Estimation Effects. To ensure accurate reliability results (that is, $\varepsilon_{P f}$ values below $10 \%$ ), the convergence metrics of the $P_{f}$ were analyzed and displayed in Fig. 13. Figure $13(a)$ depicts the $\varepsilon_{P f}$ of the $P_{f}^{T}$ used in the industry as a function of the number of samples. In industry typical, $P_{f}^{T}$ for support structures are $1 \times 10^{-3}$ or $1 \times 10^{-4}$ in more conservative cases, since the failure of the OWT support structure may not produce human damage or severe environmental damage $[39,40]$. Hence, from Fig. 13(a) the minimum number of samples $N_{P}$ that is necessary to yield an $\varepsilon_{P f}$ below $10 \%$ for both target probabilities is $4 \times 10^{6}$. Nevertheless, after running the analysis, for the default and reduced cases, using $4 \times 10^{6}$ samples, it was found that the $P_{f}$ of the structure for an operational life of 30 years was around $1 \times 10^{-6}$ with a $\delta_{P f}$ over $60 \%$. To reduce the high values of $\delta_{P f}$, and to investigate the influence of the $N_{P}$ in the $P_{f}$ more simulations were conducted, for the default and reduced cases, which the results can be seen in Fig. 13(b). From the plots, it is apparent that after $8 \times 10^{6}$ samples, not $4 \times 10^{6}$, the $P_{f}$ presents a steady behavior, thus convergence. At the same time, values of $\delta_{P f}$ of $35 \%$, and the $\varepsilon_{P f}$ of 3.5 and $7.1 \%$ for the two respective $P_{f}^{T}$, were found, which in turn are significantly lower than the initial estimation

Besides, the results of the mean-square convergence criterion for $8 \times 10^{6}$ samples of $D_{30}$ generated with MC during the processing step are shown in Fig. 14. From the graphs, it is observed that after $10^{4}$ samples the RMSE presents negligible values, less than $1 \times 10^{-3}$, and the mean estimator presents a steady behavior. The standard deviation (STD) estimator, instead, tends to converge at a slightly higher number of samples, greater than $5 \times 10^{4}$ samples; despite that, the three estimators computed out the samples show 


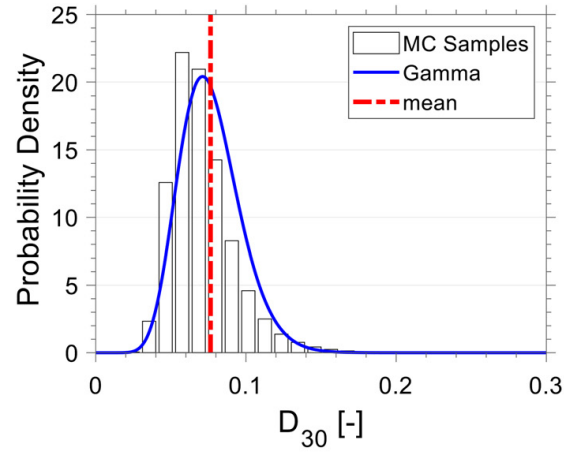

(a)

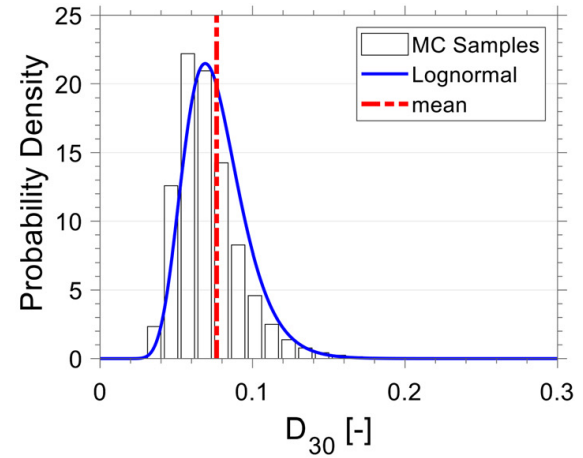

(b)

Fig. 15 Probabilistic results comparison for the OWT fatigue damage: (a) gamma PFD and (b) lognormal PDF

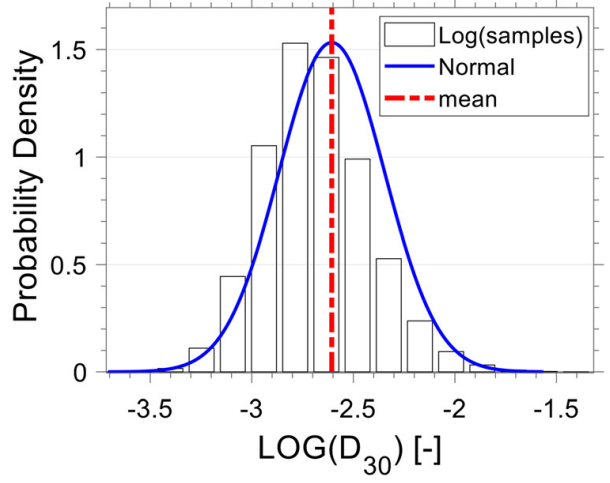

Fig. 16 Natural log of the structural damage for the OWT fatigue damage

a relatively steady behavior after $10^{5}$ samples, which in turn ensure the convergence of samples from MC. As a result, the samples obtained can be used as reliable information to determine the statistical information of the response, that is, statistical moments and PDFs from the default and reduce cases during the postprocessing step $[33,34]$.

Finally, an $N_{P}$ value of $8 \times 10^{6}$ was selected as the optimum, since it does not only meet the mean-square convergence criterion but also provides low error and variability values related to the $P_{f}$ Consequently, the selected $N_{P}$ ensured the robustness of the estimations obtained in this work, as well as reduces the need for extra sampling considering that values above $8 \times 10^{6}$ would not yield a significant gain in the accuracy of $P_{f}$ estimations.

3.3.2 Uncertainty Quantification Modeling Impacts on Fatigue Life Prediction. In this section, the application of the UQ scheme to select the appropriate distribution to model the randomness of $D_{30}$, and to analyze the shortcomings of a simplified UQ analysis are discussed.

To begin with, the results of the suggested probabilistic model to model the randomness of the structural damage, $D_{30}$, of the OWT under fatigue loading conditions are shown in Figs. 15 and 16. The proposed model was determined using the MaxEnt to ensure the least bias PDF for a set of constraints given by the physics of the problem. The first constrain refers to the limits of the density that are dictated by the domain of the damage, which has to be strictly positive $(\mathbb{R}+)$ and starting from zero; the second constrain refers to the scattering of fatigue experiments, which arise from the fact that its variability is finite, $\mathrm{E}\left[(X-\mu)^{2}\right]=c \&$ $c<\infty$, and tends to cluster around the mode and the mean of the histogram of the data; the third constrain is related to the tails, in which both tails, left and right, must decay converging to zero at a constant $d$, with $d<\infty$, respectively; and the last constrain is related to the positive skewness, i.e., longer right tail, observed in experimental fatigue data, $\mathrm{E}\left[((X-\mu) / \sigma)^{3}\right]=m \& 0<m<\infty$, which is why structural damage is usually modeled as lognormal or Weibull [41].

Two parametric distributions fulfilled the previous conditions that are the Gamma and Lognormal distribution since both have, (1) positive support and mean value, (2) finite variance, and (3)

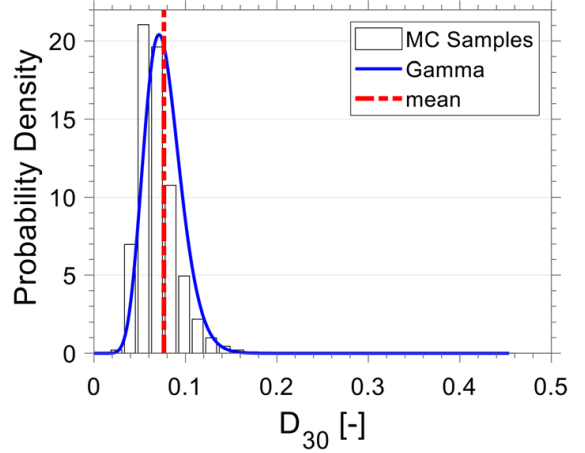

(a)

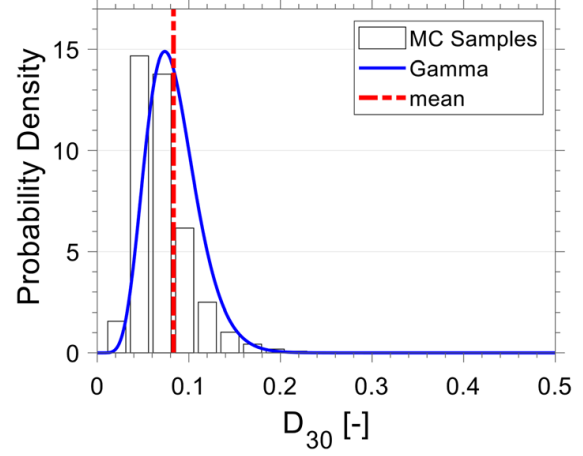

(b)

Fig. 17 Probability density functions of the accumulated damage on the OWT structure over 30 years (D30) and the resulting probability of failure for different scenarios for the available information for the most sensitive input parameters: (a) default information scenario and $(b)$ reduced information scenario 
Table 5 Statistics of the PDFs of the accumulated damage over 30 years of operation $\left(D_{30}\right)$ for the default and reduced information scenarios

\begin{tabular}{lccc}
\hline \hline & Default scenario & Reduced scenario & Variation (\%) \\
\hline Mean & 0.0764 & 0.0833 & 8.3 \\
STD & 0.021 & 0.031 & 32.3 \\
$\delta_{\mathrm{X}}$ & 0.28 & 0.376 & 25.5 \\
Range & {$[0.0240 .45]$} & {$[0.0211 .24]$} & - \\
$\mathrm{P}_{\mathrm{f}}$ & $<1 \times 10^{-7}$ & $1.2 \times 10^{-6}$ & - \\
\hline \hline
\end{tabular}

positive skewness. Figure 15 depicts both parametric models, gamma and log-normal PDF, for the 30-year damage of the OWT structure, $D_{30}$, in which their goodness of fit was assessed using the KS goodness of fit test at a $0.1 \%$ significance level; and the hyper-parameters of the distributions, scale and shape parameters, were determined using the maximum likelihood estimator for $8 \times 10^{6}$ samples drawn from MC [29].

From the figures it can be seen that there is no apparent difference between the parametric models; nevertheless, as it was pointed out in [29], a random variable is a lognormal variable only if the natural log of such random variable follows a normal distribution-that is, $\log [X] \sim N(\mu, \sigma)$. To analyze the suitability of the lognormal fit, the plot of the natural $\log$ of the $D_{30}$ was calculated and displayed in Fig. 16. As can be seen from the figure, the data is slightly skewed to the right; hence, the data have different values for the mean, mode, and median, which in turn violate the properties of a Gaussian distribution [42]. Therefore, considering that the proposed Gamma model (1) fulfilled the physical constraints of the problem with the minimum bias and (2) provided a good fit for the synthetic data at a highly significant level, the

Table 6 Information about the reduced case

\begin{tabular}{|c|c|c|c|c|}
\hline RV & Data-driven & Physics- informed & MaxEnt & References \\
\hline$\rho_{\text {air }}$ & $\begin{array}{l}\text { Supp }=[1.146-1.315] \\
\mathrm{E}[X]=1.27 \\
\delta_{X}=3[2-4] \%\end{array}$ & $\begin{array}{l}\text { Supp }=\mathbb{R}^{+} \\
\mathrm{E}[X]=m, \\
0<m<+\infty \\
\mathrm{E}\left[X^{-2}\right]=c, c<+\infty \\
\mathrm{E}[\log (X)]=d, d<+\infty\end{array}$ & Gamma & Theoretical $[7,22,27]$ \\
\hline$\alpha$ & $\begin{array}{l}\mathrm{E}[X]=0.14 \\
\delta_{X}=10 \%\end{array}$ & $\begin{array}{l}\text { Supp }=\mathbb{R}^{+} \\
\mathrm{E}[X]=m, 0<m<+\infty \\
\mathrm{E}\left[X^{-2}\right]=c, c<+\infty \\
\mathrm{E}[\log (X)]=d, d<+\infty\end{array}$ & Gamma & Theoretical $[7,21,27]$ \\
\hline$U_{10}$ & $\begin{array}{l}\mathrm{E}[X]=U_{10}(\text { state }) \\
\mathrm{E}\left[(X-\mu)^{2}\right]=\left(\sigma_{\mathrm{NTM}}\right)^{2}\end{array}$ & $\begin{array}{l}\operatorname{Supp}=\mathbb{R}^{+} \\
\mathrm{E}\left[X^{-2}\right]=c, c<+\infty \\
\mathrm{E}[\log (X)]=d, d<+\infty \\
\mathrm{E}\left[(\log (X)-\mu)^{2}\right]=m, \\
0<m<+\infty\end{array}$ & Log-normal & Data [25] \\
\hline$\rho_{\mathrm{sw}}$ & $\begin{array}{l}\text { Supp }=[1020-1030] \\
\mathrm{E}[X]=1027 \mathrm{~kg} / \mathrm{m}^{3} \\
\delta_{X}=1[0-2] \%\end{array}$ & $\begin{array}{l}\mathrm{Supp}=\mathbb{R}^{+} \\
\mathrm{E}[X]=m, 0<m<+\infty \\
\mathrm{E}\left[X^{-2}\right]=c, c<+\infty \\
\mathrm{E}[\log (X)]=d, d<+\infty\end{array}$ & Gamma & Theoretical $[22,28]$ \\
\hline$C_{m}$ & $\begin{array}{l}\text { Supp }=[1.7-2.15] \\
\mathrm{E}[X]=2 \\
\delta_{X}=10[6-30] \%\end{array}$ & Supp $=[a, b]$ & Uniform & Theoretical $[21,22]$ \\
\hline$C_{d}$ & $\begin{array}{l}\text { Supp }=[0.7-1.2] \\
\mathrm{E}[X]=0.95 \\
\delta_{X}=10[6-25] \%\end{array}$ & $\begin{array}{l}\operatorname{Supp}=[a, b] \\
\mathrm{E}[\log (X-a)]=d \\
\mathrm{E}[\log (b-X)]=e \\
|d| \text { and }|e|<+\infty\end{array}$ & Generalized beta & Theoretical [21,22] \\
\hline$H_{S}$ & $\begin{array}{l}\mathrm{E}[X]=H_{S}(\text { state }) \\
\delta_{X}=15 \%\end{array}$ & $\begin{array}{l}\mathrm{Supp}=\mathbb{R}^{+} \\
\mathrm{E}[X]=m, 0<m<+\infty \\
\mathrm{E}\left[X^{-2}\right]=c, c<+\infty \\
\mathrm{E}[\log (X)]=d, d<+\infty\end{array}$ & Gamma & Data [25] \\
\hline$T_{Z}$ & $\begin{array}{l}\mathrm{E}[X]=T z(\text { state }) \\
\delta_{X}=15 \%\end{array}$ & $\begin{array}{l}\mathrm{Supp}=\mathbb{R}^{+} \\
\mathrm{E}[X]=m, 0<m<+\infty \\
\mathrm{E}\left[X^{-2}\right]=c, c<+\infty \\
\mathrm{E}[\log (X)]=d, d<+\infty\end{array}$ & Gamma & Data [25] \\
\hline $\mathrm{E}$ & $\begin{array}{l}\text { Supp }=[189,230] \\
\mathrm{E}[X]=208 \mathrm{GPa} \\
\delta_{X}=5.4 \% \\
\mathrm{E}\left[((X-\mu) / \sigma)^{3}\right]=0.16 \\
\mathrm{E}\left[((X-\mu) / \sigma)^{4}\right]=2.68\end{array}$ & $\begin{array}{l}\text { Supp }=\mathbb{R}^{+} \\
\mathrm{E}[X]=m, 0<m<+\infty \\
\mathrm{E}\left[X^{-2}\right]=c, c<+\infty \\
\mathrm{E}[\log (X)]=d, d<+\infty\end{array}$ & Gamma & Data [29] \\
\hline$\rho_{\mathrm{ST}}$ & $\begin{array}{l}\mathrm{E}[X]=8500 \\
\delta_{X}=1[0-2] \%\end{array}$ & $\begin{array}{l}\mathrm{Supp}=\mathbb{R}^{+} \\
\mathrm{E}[X]=m, 0<m<+\infty \\
\mathrm{E}\left[X^{-2}\right]=c, c<+\infty \\
\mathrm{E}[\log (X)]=d, d<+\infty\end{array}$ & Gamma & Theoretical [17] \\
\hline$S_{N}$ & $\begin{array}{l}\text { Supp }=[0,1] \\
\mathrm{E}[X]=0.1 \\
\delta_{X}=10[5-15] \%\end{array}$ & $\begin{array}{l}\text { Supp }=[0,1] \\
\mathrm{E}[\log (X)]=d \\
\mathrm{E}[\log (1-X)]=e \\
|d| \text { and }|e|<+\infty\end{array}$ & Beta & Theoretical [26] \\
\hline$E_{\mathrm{SO}}$ & $\begin{array}{l}\text { Supp }=[4000,7500] \\
\mathrm{E}[X]=5160 \\
\delta_{X}=30[20-50] \%\end{array}$ & Supp $=[a, b]$ & Uniform & Theoretical and expert [30] \\
\hline
\end{tabular}


Gamma estimator of $D_{30}$ can be regarded as a robust approximation of the true PDF of the cumulative fatigue response for the OWT conceptual design.

Finally, to evaluate the response impacts due to simplifications in the data-driven information used in UQ analyses, a comparison between two cases considering the most sensitive variables is presented in Fig. 17 and Table 5. Figure 17(a) shows the obtained PDF for accumulated damage on the OWT structure over 30 years of operation $\left(D_{30}\right)$ for the default case in Table 3, whereas Fig. $17(b)$ shows the PDF for the reduced case in Table 6. From Fig. 17 and Table 5, there are apparent differences between the $D_{30}$ distributions for the two different scenarios; for example, the $D_{30}$ mean and a standard deviation for the reduced scenario were 0.0764 and 0.021 , respectively; whereas the $D_{30}$ mean and a standard deviation for the reduce scenario were 0.0833 and 0.031 , respectively. The results from the $D_{30}$ statistics show variations of around $8 \%$ in the mean and more than $30 \%$ in the standard deviation. Regarding the probability of failure of the structure (i.e., the probability that the accumulated damage over 30 years exceeds one), the default information scenario showed a negligible $P_{f},<1 \times 10^{-7}$, whereas the reduced information scenario showed a $P_{f}$ of $1.2 \times 10^{-6}$. Whilst both values of the $P_{f}$ are below the target probabilities $\left(1 \times 10^{-3}\right.$ and $\left.1 \times 10^{-4}\right)$ it is evident the increase of an order of magnitude between the default and reduces scenarios.

These results reveal that the reduced information scenario can potentially lead to over-estimated calculations of the probability of failure, mainly due to higher uncertainty levels embedded on the model input parameters, compared with the default information scenario. This indicates that, at least for the input parameters which have the highest impact on the model output parameters, thorough uncertainty quantification should be performed to avoid over/under-estimations of the fatigue life of the OWT structure, which in turn would result in over/under-estimations their respective costs, during the initial stages of design.

\section{Conclusion}

A UQ framework has been developed to predict, in a consistent way, the reliability and to identify the most relevant parameters of the structural design of a monopile-support structure of an OWT operating in shallow waters under fatigue loads. This UQ framework constructs the probabilistic model taking into account not only the data-driven information but also the restrictions imposed by the physics of the problem, which shapes the form of the distributions involved so as not to violate any physical law. In other words, the proposed method fuses data-driven and physics-based information into a UQ framework, in which the features and advantages of both methods are combined to provide a more systematic and consistent uncertainty model to the OWT input parameters.

On the one hand, the SA framework combining three SA approaches showed $U_{10}$ and $\rho_{\text {air }}$ were the most sensitive parameters; while $E, \rho_{\text {st }}$, and $\rho_{\text {water }}$ were the least sensitive. Consequently, considering the less-sensitive parameters as deterministic quantities would not significantly impact the system response. In contrast, using low-quality information or omitting information about the most sensitive parameters may considerably affect the fatigue life estimations. Furthermore, the reduced sensitivity of other parameters regarded as critical in the literature, such as $H_{S}$ and $E_{\mathrm{SO}}$, may be explained by the environmental conditions and characteristics of the location, as well as the type of turbine of the farm used in the study. Consequently, albeit the high sensitivity $U_{10}$ shows the suitability of the proposed SA framework for decision making since it agrees with previous studies, special attention should be played to the previous elements (i.e., location, weather, and foundation) before generalizing the results of this study to other wind farm characteristics. Therefore, the selection of the most and less sensitive parameters is a complex exercise that requires several input-model parameters, environmental conditions, and more than one sensitivity approach to yield meaningful results for decision-making purposes.

On the other hand, the systematic UQ approach developed in this study showed that uncertainty quantification and probabilistic approaches can be performed in a rather efficient and accurate manner by using generalized surrogate models developed with a DoE with a minimum ratio of ten samples per random variable. Furthermore, it was found that combining data-driven and physical-informed information in the development of the inputmodel parameter reduces the error passed to posterior stages of the UQ scheme. Hence, more consistent estimations of the statistics and PDF of the system response $D_{30}$, as well as the variability and error of the $P_{f}$ estimator, and therefore reducing the risk of overestimating/underestimating the operational life of the OWT. Finally, although the proposed UQ framework leads to more consistent predictions of the OWT structural reliability, a thorough model validation using field/experimental data of OWT fatigue life still needs to be carried out to fully assess the accuracy of the model.

\section{Nomenclature}

$c=$ horizontal distance from the neutral axis

$c_{t}=$ variable thrust coefficient

$C_{d}=$ drag coefficient

$C_{m}=$ inertia coefficient

$\mathrm{CDF}=$ cumulative density function

$\mathrm{CV}_{\mathrm{SN}}=$ coefficient of variation of S-N curve

$D=$ structural damage

$d_{\mathrm{HUB}}=$ hub height

$d_{w}=$ height from mudline to mean surface elevation

$D_{b}=$ tower diameter at the tower bottom

$D_{P}=$ pile diameter

$D_{t}=$ tower diameter at the tower top

$D_{30}=30$-year OWT structural damage

$\mathrm{DAF}=$ dynamic amplification factor

$\mathrm{DoE}=$ design of experiments

$E=$ Young's modulus

$E_{S}=$ environmental states

$E_{\mathrm{SO}}=$ Young's modulus of the soil

$\mathrm{EE}=$ elementary effects

$f_{0}=$ natural frequency of the structure

$F_{U}(t)=$ wind force at tower bottom

$F_{W}(t)=$ hydrodynamic load

$F_{\text {wd }}(t)=$ inertial component of the wave load

$F_{\text {wi }}(t)=$ drag component of the wave load

$F_{\text {D } 30}(v)=$ cumulative density function of $D_{30}$

FLT $=$ fatigue limit state

GSA $=$ global sensitivity analysis

$H_{S}=$ significant wave height

$I_{\mathrm{CM}}=$ moment of inertia

$k=$ thickness exponent

$\mathrm{KS}=$ Kolmogorov-Smirnov goodness of fit

$\log (a)=$ intercept with the $\mathrm{S}-\mathrm{N}$ axis

LHS = Latin hypercube sampling

$m=$ negative inverse slope

$M_{\mathrm{sb}}(t)=$ mudline bending moment

$M_{U}(t)=$ wind moment at tower bottom

MaxEnt $=$ maximum entropy principle

$\mathrm{MC}=$ Monte Carlo simulation

$\mathrm{MSL}=$ mean sea level

$N_{p}=$ number of samples

$n_{i}=$ number of cycles

$N_{i}=$ number of cycles of each loading block

OWT $=$ offshore wind turbine

$P_{f}=$ probability of failure

$P_{f}^{f}=$ target probability of failure

$\mathrm{PDF}=$ probability density function

$R=$ correlation coefficient

$R_{\text {OWT }}=$ reliability of the structure 


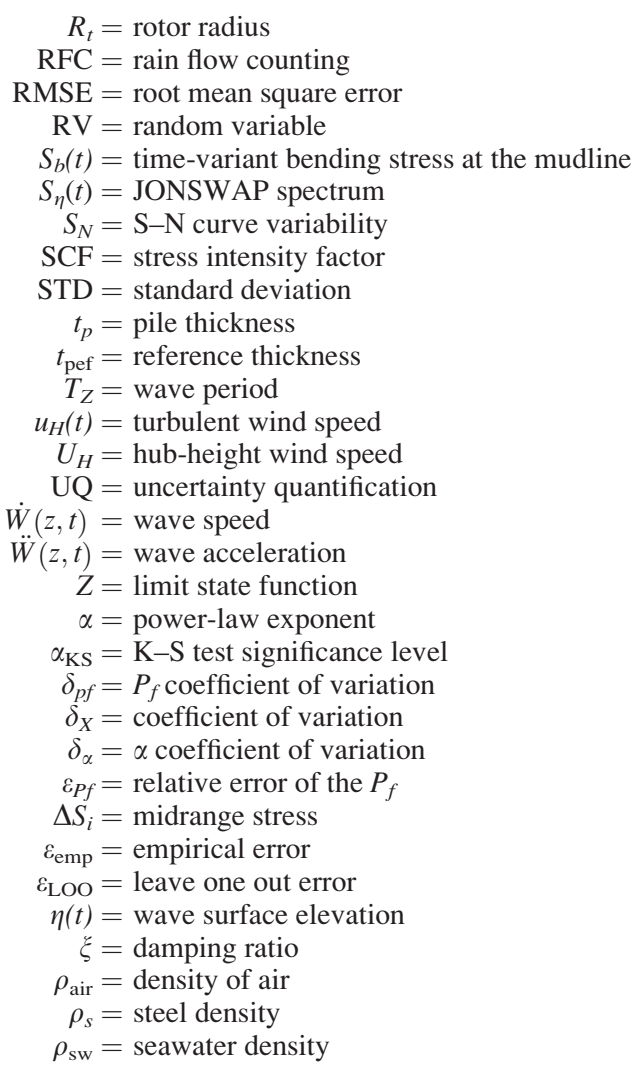

\section{References}

[1] Carswell, W., Arwade, S. R., DeGroot, D. J., and Lackner, M. A., 2015 , "Soil-Structure Reliability of Offshore Wind Turbine Monopile Foundations," Wind Energy, 18(3), pp. 483-498.

[2] Nikitas, G., Bhattacharya, S., Vimalan, N., Demirci, H. E., Nikitas, N., and Kumar, P., 2019, "Wind Power: A Sustainable Way to Limit Climate Change," Managing Global Warming, T.M. Letcher, ed., Academic Press, UK, pp. 333-364.

[3] Bisoi, S., and Haldar, S., 2014, "Dynamic Analysis of Offshore Wind Turbine in Clay Considering Soil-Monopile-Tower Interaction," Soil Dyn. Earthq. Eng., 63, pp. 19-35.

[4] Rezaei, R., Fromme, P., and Duffour, P., 2018, "Fatigue Life Sensitivity of Monopile-Supported Offshore Wind Turbines to Damping," Renew. Energy, 123, pp. 450-459.

[5] Bhattacharya, S., 2014, "Challenges in Design of Foundations for Offshore Wind Turbines," Eng. Technol. Ref., 1(1), pp. 1-9.

[6] Haldar, S., Sharma, J., and Basu, D., 2018, "Probabilistic Analysis of Monopile-Supported Offshore Wind Turbine in Clay," Soil Dyn. Earthq. Eng., 105, pp. 171-183.

[7] Toft, H. S., Svenningsen, L., Moser, W., Sørensen, J. D., and Thøgersen, M. L., 2016, "Wind Climate Parameters for WindTurbine Fatigue Load Assessment," ASME J. Sol. Energy Eng., 138(3), p. 031010.

[8] Muhammed, J. J., Jayawickrama, P. W., and Ekwaro-Osire, S., 2020 "Uncertainty Analysis in Prediction of Settlements for Spatia Prefabricated Vertical Drains Improved Soft Soil Sites," Geoscience, 10(2), p. 42.

[9] Velarde, J., Kramhøft, C., and Sørensen, J. D., 2019, "Global Sensitivity Analysis of Offshore Wind Turbine Foundation Fatigue Loads," Renew. Energy, 140(March), pp. 177-189.

[10] Pang, Z., O'Neill, Z., Li, Y., and Niu, F., 2020, "The Role of Sensitivity Analysis in the Building Performance Analysis: A Critical Review," Energy Build., 209, p. 109659.

[11] Saltelli, A., and Ratto, M., 2008, "Global Sensitivity Analysis," The Primer, Wiley, UK.

[12] Glišić, A., Ferraz, G. T., and Schaumann, P., 2017, "Sensitivity Analysis of Monopiles' Fatigue Stresses to Site Conditions Using Monte Carlo Simulation," Proceedings of the 27th International Offshore and Polar Engineering Conference, San Francisco, CA, June 25-30, pp. 305-311.

[13] Hübler, C., Gebhardt, C. G., and Rolfes, R., 2017, "Hierarchical Four-Step Global Sensitivity Analysis of Offshore Wind Turbines Based on Aeroelastic Time Domain Simulations," Renew. Energy, 111, pp. 878-891.
[14] Murcia, J. P., Réthoré, P. E., Dimitrov, N., Natarajan, A., Sørensen, J. D., Graf, P., and Kim, T., 2018, "Uncertainty Propagation Through an Aeroelastic Win Turbine Model Using Polynomial Surrogates," Renew. Energy, 119, pp. 910-922.

[15] Peeringa, J., and Bedon, G., 2017, "Fully Integrated Load Analysis Included in the Structural Reliability Assessment of a Monopile Supported Offshore Wind Turbine," Energy Procedia, 137, pp. 255-260.

[16] Nispel, A., Ekwaro-Osire, S., Dias, J. P., and Cunha Jr, A., 2019, "Probabilistic Design and Uncertainty Quantification of the Structure of a Monopile Offshore Wind Turbine," ASME Paper No. IMECE2019-11862.

[17] Jonkman, J., Butterfield, S., Musial, W., and Scott, G., 2009, Definition of a 5-MW Reference Wind Turbine for Offshore System Development, Golden, CO.

[18] Arany, L., Bhattacharya, S., MacDonald, J., and Hogan, S. J., 2017, "Design of Monopiles for Offshore Wind Turbines in 10 Steps," Soil Dyn. Earthq. Eng., 92, pp. 126-152.

[19] Bhattacharya, S., 2019, Design of Foundations for Offshore Wind Turbines, Wiley, London, UK.

[20] Jonkman, J., and Buhl, M., Jr., 2005, FAST User's Guide, Golden, CO.

[21] IEC Standard, 2009, "Wind Turbines-Part 3: Design Requirements for Offshore Wind Turbines," IEC, Geneva, Switzerland, Standard No. IEC 61400-3.

[22] DNV Standard, 2014, "Design of Offshore Wind Turbine Structures," DNV, Høvik, Norway, Standard No. DNV-OS-J101.

[23] Camp, T. R., Morris, M. J., Rooij, R., van, Tempel, J., van der, Zaaijer, M., Henderson, A., Argyriadis, K., Schwartz, S., Just, J., and Grainger, W., P., D., 2003 Design Methods for Offshore Wind Turbines at Exposed Sites, European Commission, Bristol, UK.

[24] Koukoura, C., Brown, C., Natarajan, A., and Vesth, A., 2016, "Cross-Wind Fatigue Analysis of a Full Scale Offshore Wind Turbine in the Case of WindWave Misalignment," Eng. Struct., 120, pp. 147-157.

[25] Van Der Tempel, J., 2006, Design of Support Structures for Offshore Wind Turbines, Delft University of Technology, Delft, The Netherlands.

[26] DNV Standard, 2016, "Fatigue Design of Offshore Steel Structures," DNV, Høvik, Norway, Standard No. DNVGL-RP-C203.

[27] Tamura, Y., and Kareem, A., 2013, Advanced Structural Wind Engineering, Springer, Tokyo, Japan.

[28] DNV, 2007, "Environmental Conditions and Environmental Loads," DNV, Høvik, Norway, Standard No. DNV-RP-C25, (April), pp. 1-123.

[29] Haldar, A., and Mahadevan, S., 2000, Probability, Reliability and Statistical Methods in Engineering Design, Wiley, New York.

[30] Bhattacharya, S., Wang, L., Liu, J., and Hong, Y., 2017, "Civil Engineering Challenges Associated With Design of Offshore Wind Turbines With Special Reference to China," Wind Energy Eng., pp. 243-273.

[31] Cunha Jr, A., 2017, "Modeling and Quantification of Physical Systems Uncertainties in a Probabilistic Framework," Probabilistic Prognostics and Health Management of Energy Systems, S. Ekwaro-Osire, A. C. Gonçalves, and F. M. Alemayehu, eds., Springer, Berlin, pp. 127-156.

[32] Soize, C., 2017, Uncertainty Quantification: An Accelerated Course With Advanced Applications in Computational Engineering, Springer International Publishing, Cham, Switzerland.

[33] Dias, J. P., Ekwaro-Osire, S., Cunha, A., Dabetwar, S., Nispel, A., Alemayehu, F. M., and Endeshaw, H. B., 2019, "Parametric Probabilistic Approach for Cumulative Fatigue Damage Using Double Linear Damage Rule Considering Limited Data," Int. J. Fatigue, 127, 246-258.

[34] Nispel, A., Ekwaro-Osire, S., and Dias, J. P., 2020, "Probabilistic Analysis of the Fatigue Life of Offshore Wind Turbine Structures," ASME Paper No. GT2020-14742

[35] Slot, R. M. M., Sørensen, J. D., Sudret, B., Svenningsen, L., and Thøgersen, M. L., 2020, "Surrogate Model Uncertainty in Wind Turbine Reliability Assessment," Renew. Energy, 151, pp. 1150-1162.

[36] Marelli, S., and Sudret, B., 2014, "UQLab: A Framework for Uncertainty Quantification in Matlab," Proceedings of the Second International Conference on Vulnerability and Risk Analysis and Management and the Sixth International Symposium on Uncertainty Modeling and Analysis, July 13-16, Liverpool, UK, pp. 2554-2563.

[37] Ulazia, A., Nafarrate, A, Gabriel Ibarra-Berastegi, J. S., and CarrenoMadinabeitia, S., 2019, "The Consequences of Air Density Variations Over Northeastern Scotland for Offshore Wind Energy Potential," Energies, 12 (13), 2635.

[38] Abdalla, S., and Cavaleri, L., 2002, "Effect of Wind Variability and Variable Air Density on Wave Modeling," J. Geophys. Res. C Ocean., 107(C7), p. 17.

[39] Velarde, J., Kramhøft, C., Sørensen, J. D., and Zorzi, G., 2020, "Fatigue Reliability of Large Monopiles for Offshore Wind Turbines," Int. J. Fatigue, 134, p. 105487.

[40] Velarde, J., Vanem, E., Kramhøft, C., and Sørensen, J. D., 2019, "Probabilistic Analysis of Offshore Wind Turbines Under Extreme Resonant Response: Application of Environmental Contour Method," Appl. Ocean Res., 93(October), p. 101947.

[41] Fonseca de Oliveira Correia, J. A., Jesus, A., Blasón, S., Calvente, M., and Fernández-Canteli, A., 2016, "Probabilistic Non-Linear Cumulative Fatigue Damage of the P355NL1 Pressure Vessel Steel," ASME Paper No. PVP201663920.

[42] Song, T. T., 2004, Fundamentals of Probability and Statistics for Engineers, Wiley, West Sussex, UK. 